\title{
Stability of nearly-integrable systems with dissipation
}

\author{
Alessandra Celletti \\ Dipartimento di Matematica \\ Università di Roma Tor Vergata \\ Via della Ricerca Scientifica 1 \\ I-00133 Roma (Italy) \\ (celletti@mat.uniroma2.it)
}

\author{
Christoph Lhotka \\ Dipartimento di Matematica \\ Università di Roma Tor Vergata \\ Via della Ricerca Scientifica 1 \\ I-00133 Roma (Italy) \\ (lhotka@mat.uniroma2.it)
}

November 8, 2018

\begin{abstract}
We study the stability of a vector field associated to a nearly-integrable Hamiltonian dynamical system to which a dissipation is added. Such a system is governed by two parameters, named the perturbing and dissipative parameters, and it depends on a drift function. Assuming that the frequency of motion satisfies some resonance assumption, we investigate the stability of the dynamics, and precisely the variation of the action variables associated to the conservative model. According to the structure of the vector field, one can find linear and exponential stability times, which are established under smallness conditions on the parameters. We also provide some applications to concrete examples, which exhibit a linear or exponential stability behavior.
\end{abstract}

Keywords. Dissipative systems, Stability, Resonant motion.

\section{Contents}

1 Introduction $\quad 2$

2 Notations and assumptions $\quad 4$ 
4 Applications of the normal forms 2

$4.1 \quad$ Linear stability: case $p_{X} \neq 0, s \neq 0 \ldots \ldots \ldots \ldots \ldots$

4.2 Linear stability at higher orders: case $p_{X} \neq 0, s \neq 0 \ldots \ldots \ldots 28$

4.3 Exponential stability: case $p_{X}=0, s \neq 0 \ldots \ldots \ldots \ldots$

4.4 Exponential stability: case $p_{X} \neq 0, s=0 \ldots \ldots \ldots \ldots$

5 Application of the stability estimates 32

6 Appendix A 34

6.1 Inversion of the conservative transformation . . . . . . . . . . . . 34

6.2 Non-resonance condition after the conservative transformation . . . . . . . . 34

6.3 Inversion of the dissipative transformation . . . . . . . . . . . . . 35

6.4 Non-resonance condition after the dissipative transformation . . . . . . . . 35

7 Appendix B $\quad 36$

\section{Introduction}

We investigate the behavior of nearly-integrable Hamiltonian vector fields to which a dissipative contribution is added. The vector field is ruled by two parameters, namely the perturbing parameter (measuring the non-integrability of the system) and the dissipative parameter (providing the size of the dissipative term). We assume that the phase space is contracted by time evolution. A drift function enters the equations of motion as an unknown function; its role is fundamental, since it must be properly chosen in order to meet some compatibility conditions ensuring the existence of a normal form (compare with KAM results as in [4]). We concentrate on the behavior of the variables which are actions of the conservative system (i.e. setting to zero the dissipative parameter). We assume that the initial conditions define a resonant frequency for the integrable conservative system (i.e. setting to zero both the perturbing and dissipative parameters). Under smallness conditions on the parameters, we prove that the action variables stay locally bounded over a given time interval (see also [20]). The length of the time interval depends on the functions defining the equations of motion and, precisely, whether there appear also dissipative resonant terms in the original as well as in the normalized vector field. Notice that such a result provides a useful information concerning the transient time, namely the time needed to reach the attractor.

The proof of the result is based on the construction of a suitable coordinate transformation, which is provided by the composition of a conservative and a dissipative change of variables. A similar technique, based on a non-resonant normal form, has been already implemented in 
[8] in order to investigate a vector field of the type studied in this paper, but in the simplest case of non-resonant frequency. In this case, under smallness conditions on the parameters, one can prove that the actions stay always bounded for exponential times. As in classical perturbation theory, the first transformation removes the conservative perturbation to higher orders (see, e.g., [5]); the corresponding normal form is composed by resonant or average terms. The second transformation is performed to normalize the dissipative terms; the normal form equations defining the dissipative change of variables can be solved, provided that the drift function is chosen in such a way that the compatibility condition is satisfied. The final normal form contains just resonant and average terms up to a given order in the perturbing and dissipative parameters. As in classical Nekhoroshev's theorem ([20], [21], see also [2], [13]) by properly choosing the order of the normal form, one can determine stability bounds. The stability time is exponential, whenever conservative resonant terms do not appear in the equation for the time variation of the normalized action variable or whenever the dissipative resonant contributions are zero. In the other cases the stability time depends on the inverse of the product of the perturbing and dissipative parameters. The scheme of the proof, which is presented for a non-autonomous, time-periodic system (see also [16]) follows closely [22], where a very clear and enlightening proof of Nekhoroshev's theorem is given. The proof is constructive and it allows us to provide explicit expressions for the conservative and dissipative transformations (see also [3], [10], [18]). In our opinion there are several physical problems, which can be analyzed by our method. For example, there are many results concerning the stability of the (resonant) Lagrangian points in a conservative framework (see [6], [7], [14], [15], [19]), but none of them takes into account dissipative effects (like Solar radiation, Poynting-Roberston drag, Yarkowsky effect, etc.), which might significantly affect the dynamics. In this respect, we believe that it would be interesting to analyze these models including a dissipative effect by using the results contained in this paper. We provide examples of normal forms in some concrete one-dimensional, time-dependent model problems, which illustrate different cases corresponding to linear (i.e., proportional to the inverse of the product of the perturbing and dissipative parameters) or exponential stability times. We also provide an application of the theorem in order to obtain rigorous stability bounds for the previous model problems.

The paper is organized as follows. Notations and assumptions are defined in Section 2. The resonant normal form Lemma and the stability Theorem are proven in Section 3. Examples of normal form constructions to concrete model problems is given in Section 4. An application of the stability theorem is provided in Section 5. 


\section{Notations and assumptions}

We introduce the $\ell$-dimensional, time-dependent vector field, described by the equations

$$
\begin{aligned}
& \dot{x}=\omega(y)+\varepsilon h_{10, y}(y, x, t)+\mu f_{01}(y, x, t) \\
& \dot{y}=-\varepsilon h_{10, x}(y, x, t)-\mu\left(g_{01}(y, x, t)-\eta(y, x, t)\right),
\end{aligned}
$$

where $y \in \mathbb{R}^{\ell},(x, t) \in \mathbb{T}^{\ell+1}$, while the definitions and assumptions on the parameters and functions are the following ${ }^{1}$.

1. Having fixed an initial datum $y_{0} \in \mathbb{R}^{\ell}$, we denote by $A \subset \mathbb{R}^{\ell}$ an open neighborhood of $y_{0}$.

2. The vector field depends on the parameters $\varepsilon \in \mathbb{R}_{+}$(perturbing parameter), $\mu \in \mathbb{R}_{+}$ (dissipative parameter); we remark that we could equally admit vector parameters, i.e. $\varepsilon \in \mathbb{R}_{+}^{\ell}, \mu \in \mathbb{R}_{+}^{\ell}$, but for simplicity of exposition we present the details just for the scalar case $\varepsilon \in \mathbb{R}_{+}, \mu \in \mathbb{R}_{+}$.

3. The functions $\omega$ and $\eta$ are real-analytic, $\ell$-dimensional vector functions with components $\left(\omega^{(1)}, \ldots, \omega^{(\ell)}\right)$ and $\left(\eta^{(1)}, \ldots, \eta^{(\ell)}\right)$. We assume that there exists a regular function $h_{00}=h_{00}(y)$ such that $\frac{\partial h_{00}(y)}{\partial y}=\omega(y)$. Let $h_{0}(y, u)=h_{00}(y)+u, u \in \mathbb{R}$, be the unperturbed Hamiltonian function associated to the conservative vector field $(\mu=0)$ in the extended phase space. Let $\omega_{e}(y) \equiv(\omega(y), 1)$ be the frequency vector in the extended phase space. Following [22] we make the hypothesis that $h_{0}$ is $L, M$-quasi convex, namely there exist $L, M>0$, such that for all $z \equiv(y, u) \in A \times \mathbb{R}$ at least one of the following inequalities is satisfied:

$$
\left|\omega_{e}(y) \cdot v\right|>L\|v\|, \quad \frac{\partial^{2} h_{0}(z)}{\partial z^{2}} v \cdot v \geq M\|v\|^{2}, \quad \forall v \in \mathbb{R}^{\ell+1}
$$

where the dot denotes the scalar product and $\|\cdot\|$ denotes the Euclidean norm.

4. In the following we will use the vector field (1) in the extended phase space with $\dot{t}=1$ and with $u$ conjugated to time:

$$
\begin{aligned}
& \dot{x}=\omega(y)+\varepsilon h_{10, y}(y, x, t)+\mu f_{01}(y, x, t) \\
& \dot{y}=-\varepsilon h_{10, x}(y, x, t)-\mu\left(g_{01}(y, x, t)-\eta(y, x, t)\right) \\
& \dot{u}=-\varepsilon h_{10, t}(y, x, t)+\mu \sigma(y, x, t),
\end{aligned}
$$

where the unknown function $\sigma$ is introduced for later convenience (see next point).

\footnotetext{
${ }^{1}$ The subscripts $x, y, t$ denote derivatives with respect to $x, y, t$, i.e. $h_{x} \equiv \frac{\partial h}{\partial x}, h_{y} \equiv \frac{\partial h}{\partial y}, h_{t} \equiv \frac{\partial h}{\partial t}$.
} 
5. We assume that $f_{01}, g_{01}, \eta$ are real-analytic, $\ell$-dimensional vector functions from $A \times$ $\mathbb{T}^{\ell+1}$ to $\mathbb{R}^{\ell}$, while $h_{10}, \sigma$ are periodic and real-analytic from $A \times \mathbb{T}^{\ell+1}$ to $\mathbb{R}$. We remark that $\eta, \sigma$ are unknown functions, which will be properly chosen so to meet some compatibility requirements in order to obtain a suitable normal form (see Section 3).

6. We assume that the vector field is dissipative and that the phase space volume is contracted by the time evolution.

7. For a given initial datum $y_{0}=y(0) \in A$, we assume that there exists a lattice $\Lambda \subset \mathbb{Z}^{\ell+1}$, such that the vector function $\omega=\omega\left(y_{0}\right)$ satisfies the resonance condition

$$
\left|\omega\left(y_{0}\right) \cdot k+j\right|=0 \quad \text { for all }(k, j) \in \Lambda .
$$

We also assume that there exists $K \in \mathbb{Z}_{+}, a>0$ and a subset $D \subseteq A$, such that for any $y \in D$ the following condition is satisfied:

$$
|\omega(y) \cdot k+j| \geq a \quad \text { for all }(k, j) \in \mathbb{Z}^{\ell+1} \backslash \Lambda,|k|+|j| \leq K,
$$

where for $k=\left(k_{1}, \ldots, k_{\ell}\right) \in \mathbb{Z}^{\ell}$ we define the norm $|k| \equiv\left|k_{1}\right|+\ldots+\left|k_{\ell}\right|$.

8. We refer to $\eta=\eta(y, x, t)$ as the drift vector function with components $\left(\eta^{(1)}(y, x, t), \ldots\right.$, $\left.\eta^{(\ell)}(y, x, t)\right)$ that we expand as

$$
\eta^{(k)}(y, x, t)=\sum_{m=0}^{\infty} \sum_{j=0}^{m} \eta_{j, m-j+1}^{(k)}(y, x, t) \varepsilon^{j} \mu^{m-j}, \quad k=1, \ldots, \ell .
$$

In a similar way we expand $\sigma$ in (3) as

$$
\sigma(y, x, t)=\sum_{m=0}^{\infty} \sum_{j=0}^{m} \sigma_{j, m-j+1}(y, x, t) \varepsilon^{j} \mu^{m-j} .
$$

Remark 1. We remark that for $\mu=0$ the equations (3) reduce to the conservative vector field, associated to the nearly-integrable Hamiltonian function in the extended phase space

$$
\mathcal{H}(y, x, u, t)=h_{00}(y)+u+\varepsilon h_{10}(y, x, t)
$$

where $\omega(y)=\partial h_{00}(y) / \partial y$. Notice that the Hamiltonian (6) is integrable as far as the perturbing parameter is zero, i.e. $\varepsilon=0$. Since the vector field (3) is dissipative (see assumption 6.), the energy associated to (6) is decreasing with time.

We adopt the following notations and definitions for functions and norms. 
i) Integer subscripts denote the order in the perturbing and dissipative parameters, i.e. $F_{i j}$ denotes a function of order $\varepsilon^{i} \mu^{j}$.

ii) For a function $f=f(y, x, t)$ and for any positive integer $K$, we decompose $f$ as

$$
f(y, x, t)=\bar{f}(y)+f^{(n r, \leq K)}(y, x, t)+f^{(r, \leq K)}(y, x, t)+f^{(>K)}(y, x, t),
$$

being, respectively, the average, the sum over the non-resonant components with Fourier modes less or equal than $K$, the projection over the resonant space defined by the lattice $\Lambda$ excluding the origin with Fourier modes less or equal than $K$, the sum over the components with Fourier modes greater than $K$, namely:

$$
\begin{aligned}
\bar{f}(y) & \equiv \frac{1}{(2 \pi)^{\ell+1}} \int_{\mathbb{T}^{\ell+1}} f(y, x, t) d x d t \\
f^{(n r, \leq K)}(y, x, t) & \sum_{(k, j) \in \mathbb{Z}^{\ell+1} \backslash \Lambda,|k|+|j| \leq K} \hat{f}_{k j}(y) e^{\imath(k \cdot x+j t)} \\
f^{(r, \leq K)}(y, x, t) & \sum_{(k, j) \in \Lambda \backslash\{0\},|k|+|j| \leq K} \hat{f}_{k j}(y) e^{\imath(k \cdot x+j t)} \\
f^{(>K)}(y, x, t) & \sum_{(k, j) \in \mathbb{Z}^{\ell+1},|k|+|j|>K} \hat{f}_{k j}(y) e^{\imath(k \cdot x+j t)}
\end{aligned}
$$

where $\imath=\sqrt{-1}$ and $\hat{f}_{k j}$ are the Fourier coefficients.

iii) We say that a function is of order $k$ in $\varepsilon$ and $\mu$, in symbols $O_{k}(\varepsilon, \mu)$, if its Taylor series expansion in $\varepsilon, \mu$ contains powers of $\varepsilon^{i} \mu^{j}$ with $i+j \geq k, i \geq 0, j \geq 0$.

$i v$ ) We denote by $C_{r_{0}}(A)$ the complex neighborhood of $A$ of radius $r_{0}$, namely

$$
C_{r_{0}}(A) \equiv\left\{y \in \mathbb{C}^{\ell}:\left\|y-y_{A}\right\| \leq r_{0} \text { for all } y_{A} \in A\right\}
$$

Moreover, let $C_{s_{0}}\left(\mathbb{T}^{\ell+1}\right)$ be the complex strip of radius $s_{0}$ around $\mathbb{T}^{\ell+1}$, namely

$$
C_{s_{0}}\left(\mathbb{T}^{\ell+1}\right) \equiv\left\{(x, t) \in \mathbb{C}^{\ell+1}: \max _{1 \leq j \leq \ell}\left|\Im\left(x_{j}\right)\right| \leq s_{0},|\Im(t)| \leq s_{0}\right\}
$$

where $\Im$ denotes the imaginary part.

$v$ ) Denoting the Fourier expansion of a function $f=f(y, x, t)$ as

$$
f(y, x, t)=\sum_{(k, j) \in \mathbb{Z}^{\ell+1}} \hat{f}_{k j}(y) e^{\imath(k \cdot x+j t)},
$$


we introduce the norm

$$
\|f\|_{r_{0}, s_{0}} \equiv \sup _{y \in C_{r_{0}}(A)} \sum_{(k, j) \in \mathbb{Z}^{\ell+1}}\left|\hat{f}_{k j}(y)\right| e^{(|k|+|j|) s_{0}} .
$$

For a function $g=g(y)$ we define $\|g\|_{r_{0}} \equiv \sup _{y \in C_{r_{0}}(A)}\|g(y)\|$, where $\|\cdot\|$ denotes the Euclidean norm. For a vector function $f=\left(f_{1}, . ., f_{\ell}\right)$ we define

$$
\|f\|_{r_{0}, s_{0}} \equiv \sqrt{\sum_{j=1}^{\ell}\left\|f_{j}\right\|_{r_{0}, s_{0}}^{2}}
$$

\section{Bounds on the variation of the action variables}

In order to bound the variation of the action variables, we implement a change of coordinates such that the vector field (3) is transformed to a resonant normal form, up to a suitable order $N$. To this end we introduce a change of coordinates close to the identity and leaving time unaltered, say

$$
(Y, X, U, t)=\Xi^{(N)}(y, x, u, t), \quad(Y, U) \in \mathbb{R}^{\ell+1}, \quad(X, t) \in \mathbb{T}^{\ell+1},
$$

where $\Xi^{(N)}$ depends parametrically also on $\varepsilon, \mu, \Xi^{(N)}=\Xi^{(N)}(y, x, u, t ; \varepsilon, \mu)$ with $\Xi^{(N)}(y, x, u, t ; 0,0)=$ $I d$. Let $K$ be as in (5); in the forthcoming Resonant Normal Form Lemma we aim to determine the transformation of coordinates (7), so that (3) takes a resonant normal form of order $N$, that we write as

$$
\begin{aligned}
\dot{X} & =\Omega_{d}^{(N)}(Y)+F^{(r, \leq K)}(Y, X, t)+F_{N+1}(Y, X, t)+F^{(>K)}(Y, X, t) \\
\dot{Y} & =G^{(r, \leq K)}(Y, X, t)+G_{N+1}(Y, X, t)+G^{(>K)}(Y, X, t) \\
\dot{U} & =H^{(r, \leq K)}(Y, X, t)+H_{N+1}(Y, X, t)+H^{(>K)}(Y, X, t)
\end{aligned}
$$

where $\Omega_{d}^{(N)}: \mathbb{R}^{\ell} \rightarrow \mathbb{R}^{\ell}$ is the normalized frequency, related to $\omega(Y)$ by

$$
\Omega_{d}^{(N)}(Y)=\Omega_{d}^{(N)}(Y ; \varepsilon, \mu) \equiv \omega(Y)+\sum_{i=1}^{N} \Omega_{i 0}(Y) \varepsilon^{i}+\sum_{j=1}^{N} \sum_{i=0}^{N-j} \Omega_{i j}(Y) \varepsilon^{i} \mu^{j}
$$

where $\Omega_{i j}(Y)$ are known vector functions; $F^{(r, \leq K)}, G^{(r, \leq K)}, H^{(r, \leq K)}$ have Fourier components belonging to the resonant lattice $\Lambda \backslash\{0\}$ with $F^{(r, \leq K)}$ depending on both $\varepsilon$, $\mu$, while $G^{(r, \leq K)}$, $H^{(r, \leq K)}$ depend only on $\varepsilon ; F_{N+1}, G_{N+1}, H_{N+1}$ are vector functions of order $O_{N+1}(\varepsilon, \mu)$; $F^{(>K)}, G^{(>K)}, H^{(>K)}$ denote functions with Fourier modes greater than $K$. 
Similarly to [8] we decompose the coordinate transformation $\Xi^{(N)}$ as the composition of two transformations $\Xi_{c}^{(N)}$ (conservative part) and $\Xi_{d}^{(N)}$ (dissipative part):

$$
(Y, X, U, t)=\Xi_{d}^{(N)} \circ \Xi_{c}^{(N)}(y, x, u, t) .
$$

Setting $(\tilde{y}, \tilde{x}, \tilde{u}, t) \equiv \Xi_{c}^{(N)}(y, x, u, t)$, the conservative transformation $\Xi_{c}^{(N)}$ is defined through a sequence of generating functions close to the identity, say $\psi_{j 0}=\psi_{j 0}(\tilde{y}, x, t), j=1, \ldots, N$, such that

$$
\begin{aligned}
& \tilde{x}=x+\sum_{j=1}^{N} \psi_{j 0, y}(\tilde{y}, x, t) \varepsilon^{j} \equiv x+\psi_{y}^{(N)}(\tilde{y}, x, t) \\
& y=\tilde{y}+\sum_{j=1}^{N} \psi_{j 0, x}(\tilde{y}, x, t) \varepsilon^{j} \equiv \tilde{y}+\psi_{x}^{(N)}(\tilde{y}, x, t) \\
& u=\tilde{u}+\sum_{j=1}^{N} \psi_{j 0, t}(\tilde{y}, x, t) \varepsilon^{j} \equiv \tilde{u}+\psi_{t}^{(N)}(\tilde{y}, x, t) .
\end{aligned}
$$

Notice that we can assume that the functions $\psi_{j 0}$ (as well as $\alpha_{j k}, \beta_{j k}, \gamma_{j k}$ in (12) below) do not depend on $u$, since the functions appearing in (1) (or equivalently in (3)) do not depend on $u$. We denote the inversion of (10) as

$$
\begin{aligned}
& x=x(\tilde{y}, \tilde{x}, t)=\tilde{x}+\Gamma^{(x, N)}(\tilde{y}, \tilde{x}, t) \\
& y=y(\tilde{y}, \tilde{x}, t)=\tilde{y}+\Gamma^{(y, N)}(\tilde{y}, \tilde{x}, t) \\
& u=u(\tilde{y}, \tilde{x}, t)=\tilde{u}+\Gamma^{(u, N)}(\tilde{y}, \tilde{x}, t) .
\end{aligned}
$$

The dissipative transformation $\Xi_{d}^{(N)}$ is defined by introducing suitable functions with zero average over $\tilde{x}$ and $t$, say $\alpha^{(N)}, \beta^{(N)}, \gamma^{(N)}$ defined through series by the coefficients $\alpha_{j i}, \beta_{j i}$, $\gamma_{j i}, j, i \in \mathbb{Z}_{+}$, such that

$$
\begin{aligned}
X & =\tilde{x}+\sum_{i=0}^{N} \sum_{j=0}^{i} \alpha_{j, i-j}(\tilde{y}, \tilde{x}, t) \varepsilon^{j} \mu^{i-j} \equiv \tilde{x}+\alpha^{(N)}(\tilde{y}, \tilde{x}, t) \\
Y & =\tilde{y}+\sum_{i=0}^{N} \sum_{j=0}^{i} \beta_{j, i-j}(\tilde{y}, \tilde{x}, t) \varepsilon^{j} \mu^{i-j} \equiv \tilde{y}+\beta^{(N)}(\tilde{y}, \tilde{x}, t) \\
U & =\tilde{u}+\sum_{i=0}^{N} \sum_{j=0}^{i} \gamma_{j, i-j}(\tilde{y}, \tilde{x}, t) \varepsilon^{j} \mu^{i-j} \equiv \tilde{u}+\gamma^{(N)}(\tilde{y}, \tilde{x}, t),
\end{aligned}
$$

with $\alpha_{i 0}(\tilde{y}, \tilde{x}, t)=\beta_{i 0}(\tilde{y}, \tilde{x}, t)=\gamma_{i 0}(\tilde{y}, \tilde{x}, t)=0$ for any $i \geq 0$. An iterative explicit construction of the vector functions $\psi_{j 0}, \alpha_{j i}, \beta_{j i}, \gamma_{j i}$ will be given within the proof of the Resonant Normal Form Lemma stated below. 
Remark 2. The normal form equation defining the generating function $\psi_{j 0}(\tilde{y}, \tilde{x}, t)$ at order $j$ is given by

$$
\omega(\tilde{y}) \psi_{j 0, x}(\tilde{y}, \tilde{x}, t)+\psi_{j 0, t}(\tilde{y}, \tilde{x}, t)+L_{j 0}^{(n r, \leq K)}(\tilde{y}, \tilde{x}, t)=0
$$

for a suitable known function $L_{j 0}^{(n r, \leq K)}(\tilde{y}, \tilde{x}, t)$ with zero average over $(\tilde{x}, t)$ and not containing resonant terms, say

$$
L_{j 0}^{(n r, \leq K)}(\tilde{y}, \tilde{x}, t)=\sum_{(k, j) \in \mathbb{Z}^{\ell+1} \backslash \Lambda,|k|+|j| \leq K} \hat{L}_{j 0, k j}(\tilde{y}) e^{i(k \cdot \tilde{x}+j t)} .
$$

This equation can be solved provided $\omega=\omega(\tilde{y})$ satisfies a non-resonance condition of the form

$$
\omega(\tilde{y}) \cdot k+j \neq 0 \quad \text { for all }(k, j) \in \mathbb{Z}^{\ell+1} \backslash \Lambda,|k|+|j| \leq K,
$$

which is guaranteed by (5), provided $\varepsilon$ satisfies a smallness condition. Analogously, the dissipative normal form provides an explicit construction of the functions $\alpha^{(N)}, \beta^{(N)}, \gamma^{(N)}$, thanks to a suitable choice of the drifts $\eta, \sigma$ and to the assumption (5). More precisely, once expressed in terms of the new variables $(Y, X, t)$, the functions $\beta_{j i}$ must satisfy a normal form equation of the form

$\omega(Y) \beta_{j i, x}(Y, X, t)+\beta_{j i, t}(Y, X, t)+N_{j i}^{(n r, \leq K)}(Y, X, t)+\bar{N}_{j i}(Y)+N_{j i}^{(r, \leq K)}(Y, X, t)+\eta_{j i}(Y, X, t)=0$,

for some known function $N_{j i} \equiv \bar{N}_{j i}+N_{j i}^{(n r, \leq K)}+N_{j i}^{(r, \leq K)}+N_{j i}^{(>K)}$; therefore, equation (13) can be solved provided the drift components $\eta_{j i}(Y, X, t)$ are chosen as the opposite of the sum of the average and of the resonant parts:

$$
\eta_{j i}(Y, X, t)=-\left(\bar{N}_{j i}(Y)+N_{j i}^{(r, \leq K)}(Y, X, t)\right) .
$$

An analogous relation holds for $\gamma_{j i}$ and $\sigma_{j i}$. This explains why the drift must be properly defined in order to be able to build the coordinate transformation (7). This is not unusual, but it happens also in KAM theory (see e.g.[4]). We proceed now to state the Resonant Normal Form Lemma, which extends the Normal Form Lemma of [8] to the resonant case of a frequency vector satisfying (4), (5).

Resonant Normal Form Lemma. Consider the vector field (1) analytic in the complex extension $C_{r_{0}}(A) \times C_{s_{0}}\left(\mathbb{T}^{\ell+1}\right)$ for some $r_{0}, s_{0}>0$. Consider the extended vector field (3) on $A \times \mathbb{R} \times \mathbb{T}^{\ell+1}$. For a given lattice $\Lambda \subset \mathbb{Z}^{\ell+1}$, let $y_{0} \in A, K \in \mathbb{Z}_{+}, D \subseteq A, a>0$ be such that (4) and (5) are satisfied. There exist suitable drift functions $\eta=\eta(y, x, t), \sigma=\sigma(y, x, t)$, and there exist $\varepsilon_{0}, \mu_{0}>0$ depending on $r_{0}, s_{0}, K, a$ and on the norms of $\omega, h, f, g$, such that for any $(\varepsilon, \mu) \leq\left(\varepsilon_{0}, \mu_{0}\right)$, one can construct a change of variables close to the identity, say $\Xi^{(N)}: A \times \mathbb{R} \times \mathbb{T}^{\ell+1} \rightarrow \mathbb{R}^{\ell+1} \times \mathbb{T}^{\ell+1}$ with $(Y, X, U, t)=\Xi^{(N)}(y, x, u, t)$, being $(Y, U) \in \mathbb{R}^{\ell+1}$, 
$(X, t) \in \mathbb{T}^{\ell+1}, N \in \mathbb{Z}_{+}$, which transforms (3) into a normal form of order $N$ as in (8). Let $R_{0}<r_{0}, S_{0}<s_{0}$; having set $\lambda=\max (\varepsilon, \mu)$, the normalized frequency is bounded by

$$
\left\|\Omega_{d}^{(N)}-\omega\right\|_{R_{0}} \leq C_{\omega} \lambda,
$$

where $C_{\omega}$ is a positive constant depending on $r_{0}, N$ and on the norms of $\omega, h, f, g$. Denoting by $\Pi_{y}$ the projection on the $y$-coordinate, one gets

$$
\left\|\Pi_{y}\left(\Xi_{d}^{(N)} \circ \Xi_{c}^{(N)}\right)-I d\right\| \leq C_{p} \lambda,
$$

for some positive constant $C_{p}$ depending on $r_{0}, s_{0}, N$ and on the norms of $\omega, h, f, g$. With reference to the normal form (8), one has the following estimate

$$
\left\|G^{(r, \leq K)}\right\|_{R_{0}, S_{0}}+\left\|G_{N+1}\right\|_{R_{0}, S_{0}}+\left\|G^{(>K)}\right\|_{R_{0}, S_{0}} \leq \lambda G+C_{Y} \lambda^{N+1},
$$

for some constant $C_{Y}$ and having bounded $\left\|G^{(r, \leq K)}\right\|_{R_{0}, S_{0}}$ by $\lambda G$, where $G$ and $C_{Y}$ depend on $r_{0}, s_{0}, N, K$ and on the norms of $\omega, h, f, g$. Choosing ${ }^{2} N=\left[K \tau_{0} /|\log \lambda|\right]$ for some $\tau_{0}>0$, one obtains that (16) becomes

$$
\left\|G^{(r, \leq K)}\right\|_{R_{0}, S_{0}}+\left\|G_{N+1}\right\|_{R_{0}, S_{0}}+\left\|G^{(>K)}\right\|_{R_{0}, S_{0}} \leq \lambda G+C_{Y} \lambda e^{-K \tau_{0}} .
$$

Before giving the proof of the Lemma, we provide the statement of the main result, namely a bound on the variation of the variables which are actions of the conservative system. The following Theorem will be obtained through the Resonant Normal Form Lemma under the resonance condition (4) and the quasi-convexity assumption (2). Let us write the normal form equations (8) using the following notation:

$$
\begin{aligned}
& \dot{X}=\omega(Y)+\varepsilon p_{Y}^{(\leq K)}(Y, X, t)+\mu s^{(\leq K)}(Y, X, t)+F_{N+1}(Y, X, t)+F^{(>K)}(Y, X, t) \\
& \dot{Y}=-\varepsilon p_{X}^{(\leq K)}(Y, X, t)+G_{N+1}(Y, X, t)+G^{(>K)}(Y, X, t) \\
& \dot{U}=-\varepsilon p_{t}^{(\leq K)}(Y, X, t)+H_{N+1}(Y, X, t)+H^{(>K)}(Y, X, t),
\end{aligned}
$$

where $p_{X}^{(\leq K)}, p_{Y}^{(\leq K)}, p_{t}^{(\leq K)}$ (independent of $\mu$ ) are the resonant contributions stemming just from the conservative transformation, while $s^{(\leq K)}$ (depending on $\mu$ and $\varepsilon$ ) represents the resonant part coming from the dissipative transformation.

Theorem Consider the vector field (1) defined on $A \times \mathbb{T}^{\ell+1}$, satisfying the quasi-convexity assumption (2). Let $y_{0} \in A, K \in \mathbb{Z}_{+}, D \subseteq A, a>0$ be such that (4) and (5) are satisfied. Assume there exists $\varepsilon_{0}, \mu_{0}$, such that for $(\varepsilon, \mu) \leq\left(\varepsilon_{0}, \mu_{0}\right)$, the Resonant Normal Form Lemma holds. Let $\tau_{0}, C_{p}, \lambda, r_{0}, s_{0}$ as in the Resonant Normal Form Lemma. With reference to (18), we have that:

\footnotetext{
${ }^{2}$ The choice of $N$ is motivated as follows. The relation $\lambda^{N}=e^{-K \tau_{0}}$ implies $N \log \lambda=-K \tau_{0}$, namely $N=\left[K \tau_{0} /|\log \lambda|\right]$, where $[\cdot]$ denotes the integer part.
} 
i) if $p_{X}^{(\leq K)}=0$ or $s^{(\leq K)}=0$, then there exist $\rho_{1}>0, C_{0}>0$, such that $\|y(t)-y(0)\| \leq$ $2 C_{p} \lambda+\rho_{1}$ for $t \leq T_{1} \equiv C_{0} e^{K \tau_{0}}$, where $C_{0}$ depends on $M, r_{0}, s_{0}, K, N$ and on the norms of $\omega, h, f, g$, while $\rho_{1}$ depends on the above and on $m, \lambda, \Lambda$;

ii) if $p_{X}^{(\leq K)} \neq 0$ and $s^{(\leq K)} \neq 0$, then there exist $\rho_{2}>0, C_{0}^{\prime}>0, C_{0}^{\prime \prime}>0$, such that if $t \leq T_{2} \equiv \min \left(C_{0}^{\prime} e^{K \tau_{0}}, \frac{C_{0}^{\prime \prime}}{\varepsilon \mu}\right)$, then $\|y(t)-y(0)\| \leq 2 C_{p} \lambda+\rho_{2}$, where $C_{0}^{\prime}, C_{0}^{\prime \prime}$ depend on $M, r_{0}, s_{0}, K, N$ and on the norms of $\omega, h, f, g$, while $\rho_{2}$ depends on the above and on $m, \lambda, \Lambda$.

Remark 3. The above theorem is stated in terms of the functions $p_{X}^{(\leq K)}$ and $s^{(\leq K)}$ appearing in the normal form equations (18); in order to decide which of the conditions $i$ ) or $i i$ ) of the Theorem is satisfied, one needs to know the explicit expression of the functions $f_{01}, g_{01}, h_{10}$ appearing in the vector field (1), tracing the resonant terms which could generate $p_{X}^{(\leq K)}$ and $s^{(\leq K)}$ by means of an explicit construction of the normal form or by means of a tree algorithm (see, e.g., [9], [11], [17] and references therein).

Remark 4. The Theorem states that in the non-resonant case (compare with [8]), as well as whenever the dissipative contribution to the resonant normal form is zero (at least up to the normalization order), one finds a variation of the actions on exponential times; otherwise, there appears a fast drift of the actions on linear (in $\varepsilon \mu$ ) times.

Proof of the Resonant Normal Form Lemma. By induction on the normalization order we prove that we can construct a normal form of type (8) by means of suitable transformations as in (10) and (12). First we prove the statement by constructing the first order normal form using the conservative and then the dissipative transformation; next, we proceed to construct the conservative and dissipative transformations at the order $N$. For sake of clarity, we split the proof into four separate steps, referring, respectively, to the first order conservative and dissipative normal forms, and to the $N$-th order conservative and dissipative transformations. Since the conservative transformation is standard, we omit some details.

Step 1: Conservative transformation for $N=1$.

We start by implementing the first order transformation

$$
\begin{aligned}
\tilde{x} & =x+\varepsilon \psi_{10, y}(\tilde{y}, x, t) \\
y & =\tilde{y}+\varepsilon \psi_{10, x}(\tilde{y}, x, t) \\
u & =\tilde{u}+\varepsilon \psi_{10, t}(\tilde{y}, x, t),
\end{aligned}
$$

where $\psi_{10}=\psi_{10}(\tilde{y}, x, t)$ is an unknown function. Let $\tilde{r}_{0}<r_{0}, \delta_{0}<s_{0}, \tilde{s}_{0} \equiv s_{0}-\delta_{0}$; then we can invert (19) as

$$
x=\tilde{x}+\varepsilon \Gamma^{(x, 1)}(\tilde{y}, \tilde{x}, t)
$$




$$
\begin{aligned}
& y=\tilde{y}+\varepsilon \Gamma^{(y, 1)}(\tilde{y}, \tilde{x}, t) \\
& u=\tilde{u}+\varepsilon \Gamma^{(u, 1)}(\tilde{y}, \tilde{x}, t),
\end{aligned}
$$

for suitable functions $\Gamma^{(x, 1)}, \Gamma^{(y, 1)}$ and $\Gamma^{(u, 1)}$, provided the following smallness condition on the parameters is satisfied (compare with Appendix A):

$$
70\left\|\psi_{10, y}\right\|_{\tilde{r}_{0}, s_{0}} e^{2 s_{0}} \delta_{0}^{-1} \varepsilon<1 .
$$

Using (19) and (3), we obtain that the conservative normal form is achieved whenever one can determine $\psi_{10}(\tilde{y}, \tilde{x}, t)$ such that

$$
\begin{aligned}
\omega_{y}(\tilde{y}) \psi_{10, x}(\tilde{y}, \tilde{x}, t)+\omega(\tilde{y}) \psi_{10, y x}(\tilde{y}, \tilde{x}, t)+\psi_{10, y t}(\tilde{y}, \tilde{x}, t)+h_{10, y}^{(n r, \leq K)}(\tilde{y}, \tilde{x}, t) & =0 \\
\omega(\tilde{y}) \psi_{10, x x}(\tilde{y}, \tilde{x}, t)+\psi_{10, x t}(\tilde{y}, \tilde{x}, t)+h_{10, x}^{(n r, \leq K)}(\tilde{y}, \tilde{x}, t) & =0 \\
\omega(\tilde{y}) \psi_{10, t x}(\tilde{y}, \tilde{x}, t)+\psi_{10, t t}(\tilde{y}, \tilde{x}, t)+h_{10, t}^{(n r, \leq K)}(\tilde{y}, \tilde{x}, t) & =0 .
\end{aligned}
$$

Let us define

$$
\Omega_{c}^{(1)}(\tilde{y})=\Omega_{c}^{(1)}(\tilde{y} ; \varepsilon) \equiv \omega(\tilde{y})+\varepsilon \bar{h}_{10, y}(\tilde{y}) ;
$$

Equations (21) are equivalent to take the derivatives with respect to $y, x$ and $t$ of

$$
\omega(\tilde{y}) \psi_{10, x}(\tilde{y}, \tilde{x}, t)+\psi_{10, t}(\tilde{y}, \tilde{x}, t)+h_{10}^{(n r, \leq K)}(\tilde{y}, \tilde{x}, t)=0 .
$$

Expanding $\psi_{10}$ and $h_{10}^{(n r, \leq K)}$ into Fourier series, one obtains that $\psi_{10}$ is given by the expression (independent of $\tilde{u}$ ):

$$
\psi_{10}(\tilde{y}, \tilde{x}, t)=\imath \sum_{(k, j) \in \mathbb{Z}^{\ell+1} \backslash \Lambda,|k|+|j| \leq K} \frac{\hat{h}_{10, k j}^{(n r, \leq K)}(\tilde{y})}{\omega(\tilde{y}) \cdot k+j} e^{\imath(k \cdot \tilde{x}+j t)} .
$$

This function is well defined, since the zero and small divisors are controlled as follows. The second of (19) can be inverted as $\tilde{y}=y+\varepsilon R^{(y, 1)}(y, x, t)$ for a suitable function $R^{(y, 1)}=$ $R^{(y, 1)}(y, x, t)$, provided that for $\tilde{r}_{0}^{\prime}<r_{0}$ one has (see Appendix A)

$$
70 \varepsilon\left\|\psi_{10, x}\right\|_{\tilde{r}_{0}, s_{0}} \frac{1}{\tilde{r}_{0}-\tilde{r}_{0}^{\prime}}<1,
$$

being $\varepsilon\left\|R^{(y, 1)}\right\|_{\tilde{r}_{0}^{\prime}, s_{0}} \leq\left\|\psi_{10, x}\right\|_{\tilde{r}_{0}, s_{0}}$. Then, the divisors appearing in (22) are bounded by

$$
|\omega(\tilde{y}) \cdot k+j| \geq a-\varepsilon K\left\|R^{(y, 1)}\right\|_{\tilde{r}_{0}^{\prime}, s_{0}}\left\|\omega_{y}\right\|_{r_{0}}>\frac{a}{2},
$$

provided

$$
\varepsilon<\frac{a}{2 K\left\|R^{(y, 1)}\right\|_{\tilde{r}_{0}^{\prime}, s_{0}}\left\|\omega_{y}\right\|_{r_{0}}} .
$$


Step 2: Dissipative transformation for $N=1$.

We define the first-order dissipative transformation as

$$
\begin{aligned}
X & =\tilde{x}+\alpha_{01}(\tilde{y}, \tilde{x}, t) \mu \\
Y & =\tilde{y}+\beta_{01}(\tilde{y}, \tilde{x}, t) \mu \\
U & =\tilde{u}+\gamma_{01}(\tilde{y}, \tilde{x}, t) \mu,
\end{aligned}
$$

for unknown functions $\alpha_{01}, \beta_{01}$ and $\gamma_{01}$. Let us start by inverting (26) as

$$
\begin{aligned}
\tilde{x} & =X-\alpha_{01}(Y, X, t) \mu+O_{2}(\mu)=X+\Delta^{(x, 1)}(Y, X, t) \mu \\
\tilde{y} & =Y-\beta_{01}(Y, X, t) \mu+O_{2}(\mu)=Y+\Delta^{(y, 1)}(Y, X, t) \mu \\
\tilde{u} & =U-\gamma_{01}(Y, X, t) \mu+O_{2}(\mu)=U+\Delta^{(u, 1)}(Y, X, t) \mu
\end{aligned}
$$

for suitable functions $\Delta^{(x, 1)}, \Delta^{(y, 1)}$ and $\Delta^{(u, 1)}$ provided the following smallness conditions on the parameters are satisfied (see Appendix A):

$$
\begin{array}{r}
70\left\|\alpha_{01}\right\|_{\tilde{r}_{0}, \tilde{s}_{0}} e^{2 \tilde{s}_{0}} \tilde{\delta}_{0}^{-1} \mu<1 \\
70\left(\left\|\beta_{01}\right\|_{\tilde{r}_{0}, \tilde{s}_{0}}+\left\|\beta_{01, x}\right\|_{\tilde{r}_{0}, \tilde{s}_{0}}\left\|\alpha_{01}\right\|_{\tilde{r}_{0}, \tilde{s}_{0}}\right) \frac{1}{\tilde{r}_{0}-R_{0}} \mu<1 \\
70\left(\left\|\gamma_{01}\right\|_{\tilde{r}_{0}, \tilde{s}_{0}}+\left\|\gamma_{01, x}\right\|_{\tilde{r}_{0}, \tilde{s}_{0}}\left\|\alpha_{01}\right\|_{\tilde{r}_{0}, \tilde{s}_{0}}\right) \frac{1}{\tilde{r}_{0}-R_{0}} \mu<1,
\end{array}
$$

where $\tilde{\delta}_{0} \equiv \tilde{s}_{0} / 2, R_{0}<\tilde{r}_{0}$ and being $\left\|\Delta^{(x, 1)}\right\|_{\tilde{r}_{0}, \tilde{s}_{0}-\tilde{\delta}_{0}} \leq\left\|\alpha_{01}\right\|_{\tilde{r}_{0}, \tilde{s}_{0}}$. Through (26) and (27) we can express $\dot{X}, \dot{Y}$ as a function of $X, Y$; the normal form is obtained assuming that $\alpha_{01}, \beta_{01}$ and $\eta_{01}$ satisfy the following equations:

$$
\begin{aligned}
\omega(Y) \alpha_{01, x}(Y, X, t) & +\alpha_{01, t}(Y, X, t)-\omega_{y}(Y) \beta_{01}(Y, X, t)+f_{01}^{(n r, \leq K)}(Y, X, t)=0 \\
\omega(Y) \beta_{01, x}(Y, X, t) & +\beta_{01, t}(Y, X, t) \\
& -g_{01}^{(n r, \leq K)}(Y, X, t)-\bar{g}_{01}(Y)-g_{01}^{(r, \leq K)}(Y, X, t)+\eta_{01}(Y, X, t)=0 \\
\omega(Y) \gamma_{01, x}(Y, X, t) & +\gamma_{01, t}(Y, X, t)+\sigma_{01}(Y, X, t)=0 .
\end{aligned}
$$

Since $\alpha_{i j}, \beta_{i j}, \gamma_{i j}$ have zero average and they do not contain resonant terms, the system of equations (29) can be solved, provided that we choose $\eta_{01}(Y, X, t)$ as

$$
\eta_{01}(Y, X, t) \equiv \bar{g}_{01}(Y)+g_{01}^{(r, \leq K)}(Y, X, t)
$$

and that we set $\sigma_{01}=0$ as well as $\gamma_{01}=0$. Setting $\Omega_{d}^{(1)}=\omega(Y)+\varepsilon \bar{h}_{10, y}(Y)+\mu \bar{f}_{01}(Y)$, the first order normal form can be written as

$$
\begin{aligned}
\dot{X} & =\Omega_{d}^{(1)}+\varepsilon h_{10, y}^{(r, \leq K)}(Y, X, t)+\mu f_{01}^{(r, \leq K)}(Y, X, t) \\
& +\varepsilon h_{10, y}^{(>K)}(Y, X, t)+\mu f_{01}^{(>K)}(Y, X, t)+F_{2}(Y, X, t) \\
\dot{Y} & =-\varepsilon h_{10, x}^{(r, \leq K)}(Y, X, t)-\varepsilon h_{10, x}^{(>K)}(Y, X, t)-\mu g_{01}^{(>K)}(Y, X, t)+G_{2}(Y, X, t) \\
\dot{U} & =-\varepsilon h_{10, t}^{(r, \leq K)}(Y, X, t)-\varepsilon h_{10, t}^{(>K)}(Y, X, t)+H_{2}(Y, X, t),
\end{aligned}
$$


where $F_{2}, G_{2}$ are functions of order $\mathrm{O}_{2}(\varepsilon, \mu)$ and $\mathrm{H}_{2}$ is a function of order $\mathrm{O}_{2}(\varepsilon)$. We remark that the solution of (29) involves small divisors of the form $\omega(Y) \cdot k+j$ with $(k, j) \in \mathbb{Z}^{\ell+1}$ and $|k|+|j| \leq K$. Using the same argument as in (24), the small divisors are bounded by $a / 4$ provided that the following smallness condition holds (compare with Appendix A):

$$
\mu<\frac{a}{4 K\left\|\beta_{01}\right\|_{\tilde{r}_{0}, \tilde{s}_{0}}\left\|\omega_{y}\right\|_{r_{0}}} .
$$

\section{Step 3: Conservative transformation for the order $N$.}

Assume that the Lemma holds to the order $N-1$. We introduce the conservative transformation to the $\operatorname{order} N$ as

$$
\begin{aligned}
& \tilde{x}=x+\sum_{j=1}^{N-1} \psi_{j 0, y}(\tilde{y}, x, t) \varepsilon^{j}+\psi_{N 0, y}(\tilde{y}, x, t) \varepsilon^{N} \equiv x+\psi_{y}^{(N)}(\tilde{y}, x, t) \\
& y=\tilde{y}+\sum_{j=1}^{N-1} \psi_{j 0, x}(\tilde{y}, x, t) \varepsilon^{j}+\psi_{N 0, x}(\tilde{y}, x, t) \varepsilon^{N} \equiv \tilde{y}+\psi_{x}^{(N)}(\tilde{y}, x, t) \\
& u=\tilde{u}+\sum_{j=1}^{N-1} \psi_{j 0, t}(\tilde{y}, x, t) \varepsilon^{j}+\psi_{N 0, t}(\tilde{y}, x, t) \varepsilon^{N} \equiv \tilde{u}+\psi_{t}^{(N)}(\tilde{y}, x, t),
\end{aligned}
$$

where for $1 \leq j \leq N-1$ the functions $\psi_{j 0}(\tilde{y}, x, t)$ are assumed to be known. We can invert (31) as

$$
\begin{aligned}
x & =x(\tilde{y}, \tilde{x}, t) \\
& =\tilde{x}+\sum_{j=1}^{N} \Gamma_{j 0}^{(x)}(\tilde{y}, \tilde{x}, t) \varepsilon^{j}-\psi_{N 0, y}(\tilde{y}, \tilde{x}, t) \varepsilon^{N}+O_{N+1}(\varepsilon) \equiv \tilde{x}+\Gamma^{(x, N)}(\tilde{y}, \tilde{x}, t) \\
y & =y(\tilde{y}, \tilde{x}, t) \\
& =\tilde{y}+\sum_{j=1}^{N} \Gamma_{j 0}^{(y)}(\tilde{y}, \tilde{x}, t) \varepsilon^{j}+\psi_{N 0, x}(\tilde{y}, \tilde{x}, t) \varepsilon^{N}+O_{N+1}(\varepsilon) \equiv \tilde{y}+\Gamma^{(y, N)}(\tilde{y}, \tilde{x}, t) \\
u & =u(\tilde{y}, \tilde{x}, t) \\
& =\tilde{u}+\sum_{j=1}^{N} \Gamma_{j 0}^{(u)}(\tilde{y}, \tilde{x}, t) \varepsilon^{j}+\psi_{N 0, t}(\tilde{y}, \tilde{x}, t) \varepsilon^{N}+O_{N+1}(\varepsilon) \equiv \tilde{u}+\Gamma^{(u, N)}(\tilde{y}, \tilde{x}, t),
\end{aligned}
$$

provided that (see Appendix A), choosing $\tilde{r}_{0}<r_{0}, \delta_{0}<s_{0}$ :

$$
70\left\|\psi_{y}^{(N)}\right\|_{\tilde{r}_{0}, s_{0}} e^{2 s_{0}} \delta_{0}^{-1}<1
$$


being $\Gamma_{j 0}^{(x)}, \Gamma_{j 0}^{(y)}, \Gamma_{j 0}^{(u)}, 1 \leq j \leq N$, known functions. We proceed to compute $\dot{x}, \dot{y}, \dot{u}$ as a function of $\tilde{x}, \tilde{y}, t$ and after expanding in Taylor series, we obtain

$$
\begin{aligned}
\dot{x} & =\omega(\tilde{y})+\omega_{y}(\tilde{y}) \psi_{N 0, x}(\tilde{y}, \tilde{x}, t) \varepsilon^{N}+F^{(1, \leq K, \leq N)}(\tilde{y}, \tilde{x}, t)+O_{N+1}^{>K} \\
\dot{y} & =G^{(1, \leq K, N)}(\tilde{y}, \tilde{x}, t)+\mu\left(\eta_{N-1,1}(\tilde{y}, \tilde{x}, t) \varepsilon^{N-1}+\ldots+\eta_{0, N}(\tilde{y}, \tilde{x}, t) \mu^{N-1}\right)+O_{N+1}^{>K} \\
\dot{u} & =H^{(1, \leq K, N)}(\tilde{y}, \tilde{x}, t)+\mu\left(\sigma_{N-1,1}(\tilde{y}, \tilde{x}, t) \varepsilon^{N-1}+\ldots+\sigma_{0, N}(\tilde{y}, \tilde{x}, t) \mu^{N-1}\right)+O_{N+1}^{>K}
\end{aligned}
$$

where $O_{N+1}^{>K}$ is a compact notation to denote terms of order $O_{N+1}(\varepsilon, \mu)$ and/or containing Fourier components greater than $K$; the functions $F^{(1, \leq K, \leq N)}, G^{(1, \leq K, N)}, H^{(1, \leq K, N)}$ are known, contain Fourier components up to the order $K$, contain orders in $\varepsilon$ and $\mu$ up to the order $N$ and they are at most linear in $\mu$. Using (31), (34) and the inductive hypothesis, the conservative normal form at the order $N$ is obtained once the function $\psi_{N 0}$ satisfies the equations

$$
\begin{aligned}
\omega_{y}(\tilde{y}) \psi_{N 0, x}(\tilde{y}, \tilde{x}, t)+\omega(\tilde{y}) \psi_{N 0, y x}(\tilde{y}, \tilde{x}, t)+\psi_{N 0, y t}(\tilde{y}, \tilde{x}, t)+F_{N 0}^{(2, n r, \leq K, \leq N)}(\tilde{y}, \tilde{x}, t) & =0 \\
\omega(\tilde{y}) \psi_{N 0, x x}(\tilde{y}, \tilde{x}, t)+\psi_{N 0, x t}(\tilde{y}, \tilde{x}, t)-G_{N 0}^{(2, n r, \leq K, \leq N)}(\tilde{y}, \tilde{x}, t) & =0 \\
\omega(\tilde{y}) \psi_{N 0, t x}(\tilde{y}, \tilde{x}, t)+\psi_{N 0, t t}(\tilde{y}, \tilde{x}, t)-H_{N 0}^{(2, n r, \leq K, \leq N)}(\tilde{y}, \tilde{x}, t) & =0,
\end{aligned}
$$

where $F_{N 0}^{(2, n r, \leq K, \leq N)}, G_{N 0}^{(2, n r, \leq K, \leq N)}, H_{N 0}^{(2, n r, \leq K, \leq N)}$ are the non-resonant parts of known functions $F^{(2, \leq K, \leq N)}, G^{(2, \leq K, \leq N)} H^{(2, \leq K, \leq N)}$ that we decompose as

$$
\begin{aligned}
F^{(2, \leq K, \leq N)}(\tilde{y}, \tilde{x}, t) & \equiv \sum_{j=1}^{N} \bar{F}_{j 0}^{(2, \leq N)}(\tilde{y}) \varepsilon^{j}+\sum_{j=1}^{N} F_{j 0}^{(2, n r, \leq K, \leq N)}(\tilde{y}, \tilde{x}, t) \varepsilon^{j} \\
& +\sum_{j=1}^{N} F_{j 0}^{(2, r, \leq K, \leq N)}(\tilde{y}, \tilde{x}, t) \varepsilon^{j}+\mu \sum_{j=0}^{N-1} F_{j 1}^{(2, \leq K, \leq N)}(\tilde{y}, \tilde{x}, t) \varepsilon^{j}
\end{aligned}
$$

(and similar for the remaining functions). From the Hamiltonian structure it can be easily recognized that $F_{N 0}^{(2, n r, \leq K, \leq N)}, G_{N 0}^{(2, n r, \leq K, \leq N)}, H_{N 0}^{(2, n r, \leq K, \leq N)}$ are, respectively, the derivatives with respect to $y, x, t$ of the same function, so that equations (35) uniquely define the solution $\psi_{N 0}(\tilde{y}, \tilde{x}, t)$. We are finally led to the following conservative normal form:

$$
\begin{aligned}
& \dot{\tilde{x}}=\Omega_{c}^{(N)}(\tilde{y} ; \varepsilon)+\sum_{j=1}^{N} F_{j 0}^{(2, r, \leq K, \leq N)}(\tilde{y}, \tilde{x}, t) \varepsilon^{j}+\mu \sum_{j=0}^{N-1} F_{j 1}^{(2, \leq K, \leq N)}(\tilde{y}, \tilde{x}, t) \varepsilon^{j}+O_{N+1}^{>K} \\
& \dot{\tilde{y}}=\sum_{j=1}^{N} G_{j 0}^{(2, r, \leq K, \leq N)}(\tilde{y}, \tilde{x}, t) \varepsilon^{j}+\mu \sum_{j=0}^{N-1} G_{j 1}^{(2, \leq K, \leq N)}(\tilde{y}, \tilde{x}, t) \varepsilon^{j}+O_{N+1}^{>K} \\
& \dot{\tilde{u}}=\sum_{j=1}^{N} H_{j 0}^{(2, r, \leq K, \leq N)}(\tilde{y}, \tilde{x}, t) \varepsilon^{j}+\mu \sum_{j=0}^{N-1} H_{j 1}^{(2, \leq K, \leq N)}(\tilde{y}, \tilde{x}, t) \varepsilon^{j}+O_{N+1}^{>K},
\end{aligned}
$$


where

$$
\Omega_{c}^{(N)}(\tilde{y} ; \varepsilon) \equiv \omega(\tilde{y})+\sum_{j=1}^{N} \bar{F}_{j 0}^{(2, N)}(\tilde{y}) \varepsilon^{j},
$$

which implies that $\left\|\Omega_{c}^{(N)}-\omega\right\| \leq C_{c} \varepsilon$ for a suitable constant $C_{c}$. The normal form equations can be solved, provided that the small divisors taking the expression $\omega(\tilde{y}) \cdot k+j$, for $k \in \mathbb{Z}^{\ell}$, $j \in \mathbb{Z}$ with $|k|+|j| \leq K$, are controlled by a non-resonance condition, which is guaranteed whenever (see Appendix A)

$$
\varepsilon \leq \frac{a}{2 K\left\|R^{(y, N)}\right\|_{\tilde{r}_{0}^{\prime}, s_{0}}\left\|\omega_{y}\right\|_{r_{0}}}
$$

where $R^{(y, N)}$ is the function inverting the transformation, namely $\tilde{y}=y+\varepsilon R^{(y, N)}(y, x, t)$, and $\tilde{r}_{0}^{\prime}<\tilde{r}_{0}$. The inversion can be performed provided (see Appendix A)

$$
70\left\|\psi_{x}^{(N)}\right\|_{\tilde{r}_{0}, s_{0}} \frac{1}{r_{0}-\tilde{r}_{0}^{\prime}}<1
$$

with $\left\|R^{(y, N)}\right\|_{\tilde{r}_{0}^{\prime}, s_{0}} \leq\left\|\psi_{x}^{(N)}\right\|_{\tilde{r}_{0}, s_{0}}$.

\section{Step 4: Dissipative transformation for the order $N$.}

We consider the transformation (12) at the order $N$, which can be inverted as

$$
\begin{aligned}
\tilde{x} & =\tilde{x}(Y, X, t) \\
& =X+\sum_{i=0}^{N-2} \sum_{j=1}^{N-1-i} a_{i j}(Y, X, t) \varepsilon^{i} \mu^{j}-\sum_{i=0}^{N-1} \alpha_{i, N-i}(Y, X, t) \varepsilon^{i} \mu^{N-i}+O_{N+1}(\varepsilon, \mu) \\
\tilde{y} & =\tilde{y}(Y, X, t) \\
& =Y+\sum_{i=0}^{N-2} \sum_{j=1}^{N-1-i} b_{i j}(Y, X, t) \varepsilon^{i} \mu^{j}-\sum_{i=0}^{N-1} \beta_{i, N-i}(Y, X, t) \varepsilon^{i} \mu^{N-i}+O_{N+1}(\varepsilon, \mu) \\
\tilde{u} & =\tilde{u}(Y, X, t) \\
& =U+\sum_{i=0}^{N-2} \sum_{j=1}^{N-1-i} c_{i j}(Y, X, t) \varepsilon^{i} \mu^{j}-\sum_{i=0}^{N-1} \gamma_{i, N-i}(Y, X, t) \varepsilon^{i} \mu^{N-i}+O_{N+1}(\varepsilon, \mu),
\end{aligned}
$$

for suitable known functions $a_{i j}(Y, X, t), b_{i j}(Y, X, t), c_{i j}(Y, X, t)$, provided that the parameters satisfy (see Appendix A):

$$
\begin{aligned}
& 70\left\|\alpha^{(N)}\right\|_{\tilde{r}_{0}, \tilde{s}_{0}} e^{2 \tilde{s}_{0}} \tilde{\delta}_{0}^{-1}<1 \\
& 70\left(\left\|\beta^{(N)}\right\|_{\tilde{r}_{0}, \tilde{s}_{0}}+\left\|\beta_{x}^{(N)}\right\|_{\tilde{r}_{0}, \tilde{s}_{0}}\left\|\alpha^{(N)}\right\|_{\tilde{r}_{0}, \tilde{s}_{0}}\right) \frac{1}{\tilde{r}_{0}-R_{0}}<1 \\
& 70\left(\left\|\gamma^{(N)}\right\|_{\tilde{r}_{0}, \tilde{s}_{0}}+\left\|\gamma_{x}^{(N)}\right\|_{\tilde{r}_{0}, \tilde{s}_{0}}\left\|\alpha^{(N)}\right\|_{\tilde{r}_{0}, \tilde{s}_{0}}\right) \frac{1}{\tilde{r}_{0}-R_{0}}<1
\end{aligned}
$$


where $\tilde{\delta}_{0} \equiv \tilde{s}_{0} / 2, R_{0}<\tilde{r}_{0}$. In order to determine the unknown functions $\alpha_{0, N}, \ldots, \alpha_{N-1,1}$, $\beta_{0, N}, \ldots, \beta_{N-1,1}, \gamma_{0, N}, \ldots, \gamma_{N-1,1}, \eta_{N-1,0}, \ldots, \eta_{0, N-1}, \sigma_{N-1,0}, \ldots, \sigma_{0, N-1}$, using (37) and (40) we express $\dot{\tilde{x}}, \dot{\tilde{y}}$ in terms of $X, Y$ and we compute $\dot{X}, \dot{Y}$ using (12), (37), (40) as

$$
\begin{aligned}
\dot{X} & =\omega(Y)-\omega_{y}(Y)\left(\sum_{i=0}^{N-1} \beta_{i, N-i}(Y, X, t) \varepsilon^{i} \mu^{N-i}\right)+\omega(Y) \sum_{i=0}^{N-1} \alpha_{i, N-i, x}(Y, X, t) \varepsilon^{i} \mu^{N-i} \\
& +\sum_{i=0}^{N-1} \alpha_{i, N-i, t}(Y, X, t) \varepsilon^{i} \mu^{N-i}+F^{(3, n r, \leq K, N)}(Y, X, t)+\bar{F}^{(3, \leq N)}(Y) \\
& +F^{(3, r, \leq K, \leq N)}(Y, X, t)+O_{N+1}^{>K} \\
& =\mu\left(\eta_{N-1,1}(Y, X, t) \varepsilon^{N-1}+\ldots+\eta_{0, N}(Y, X, t) \mu^{N-1}\right)+\omega(Y) \sum_{i=0}^{N-1} \beta_{i, N-i, x}(Y, X, t) \varepsilon^{i} \mu^{N-i} \\
& +\sum_{i=0}^{N-1} \beta_{i, N-i, t}(Y, X, t) \varepsilon^{i} \mu^{N-i}+G^{(3, n r, \leq K, N)}(Y, X, t)+\bar{G}^{(3, N)}(Y) \\
& +\sum_{i=1}^{N} G_{i 0}^{(3, r, \leq K, \leq N)}(Y, X, t) \varepsilon^{i}+G^{(3, r, \leq K, N)}(Y, X, t)+O_{N+1}^{>K} \\
& =\mu\left(\sigma_{N-1,1}(Y, X, t) \varepsilon^{N-1}+\ldots+\sigma_{0, N}(Y, X, t) \mu^{N-1}\right)+\omega(Y) \sum_{i=0}^{N-1} \gamma_{i, N-i, x}(Y, X, t) \varepsilon^{i} \mu^{N-i} \\
& +\sum_{i=0}^{N-1} \gamma_{i, N-i, t}(Y, X, t) \varepsilon^{i} \mu^{N-i}+H^{(3, n r, \leq K, N)}(Y, X, t)+\bar{H}^{(3, N)}(Y) \\
& +\sum_{i=1}^{N} H_{i 0}^{(3, r, \leq K, \leq N)}(Y, X, t) \varepsilon^{i}+H^{(3, r, \leq K, N)}(Y, X, t)+O_{N+1}^{>K},
\end{aligned}
$$

where $F^{(3, n r, \leq K, N)}, G^{(3, n r, \leq K, N)}, H^{(3, n r, \leq K, N)}$ denote known non-resonant functions that we can expand as

$$
F^{(3, n r, \leq K, N)}(Y, X, t)=\sum_{i=0}^{N-1} F_{i, N-1}^{(3, n r, \leq K, N)}(Y, X, t) \varepsilon^{i} \mu^{N-i}
$$

and similarly for $G^{(3, n r, \leq K, N)}, H^{(3, n r, \leq K, N)} ; F^{(3, r, \leq K, \leq N)}, G^{(3, r, \leq K, N)}, H^{(3, r, \leq K, N)}$ denote known resonant functions; $\bar{F}^{(3, \leq N)}, \bar{G}^{(3, N)}, \bar{H}^{(3, N)}$ denote the average terms. Recall that due to the inductive hypothesis, the functions $\alpha_{i j}, \beta_{i j}, \gamma_{i j}, \eta_{i j}, \sigma_{i j}$, determine a normal form up to the order $\varepsilon^{i} \mu^{j}$ with $0 \leq i+j \leq N-1$. The normal form at the order $N$ is obtained by imposing that $\alpha_{i j}, \beta_{i j}, \gamma_{i j}, \eta_{i j}, \sigma_{i j}$ satisfy the normal form equations

$$
-\omega_{y}(Y) \beta_{i, N-i}(Y, X, t)+\omega(Y) \alpha_{i, N-i, x}(Y, X, t)+\alpha_{i, N-i, t}(Y, X, t)
$$




$$
\begin{gathered}
+F_{i, N-i}^{(3, n r, \leq K, N)}(Y, X, t)=0 \\
\omega(Y) \beta_{i, N-i, x}(Y, X, t)+\beta_{i, N-i, t}(Y, X, t)+G_{i, N-i}^{(3, n r, \leq K, N)}(Y, X, t)+G_{i, N-i}^{(3, r, \leq K, \leq N)}(Y, X, t) \\
+\bar{G}_{i, N-i}^{(3, N)}(Y)+\eta_{i, N-i}(Y, X, t)=0 \\
\omega(Y) \gamma_{i, N-i, x}(Y, X, t)+\gamma_{i, N-i, t}(Y, X, t)+H_{i, N-i}^{(3, n r, \leq K, N)}(Y, X, t)+H_{i, N-i}^{(3, r, \leq K, \leq N)}(Y, X, t) \\
+\bar{H}_{i, N-i}^{(3, N)}(Y)+\sigma_{i, N-i}(Y, X, t)=0
\end{gathered}
$$

for $0 \leq i \leq N-1$. Equations (42) can be solved provided $\omega(Y)$ satisfies a non-resonance condition, which is guaranteed by (see Appendix A)

$$
4 K\left\|\omega_{y}\right\|_{r_{0}}\left\|\beta^{(N)}\right\|_{\tilde{r}_{0}, \tilde{s}_{0}}<a,
$$

where we intend that $Y \equiv \tilde{y}+\beta^{(N)}(\tilde{y}, \tilde{x}, t ; \varepsilon, \mu)$. From the second and third of (42), we get

$$
\begin{aligned}
\eta_{i, N-i}(Y, X, t) & \equiv \bar{G}_{i, N-i}^{(3, N)}(Y)+G_{i, N-i}^{(3, r, \leq K, N)}(Y, X, t) \varepsilon^{i} \mu^{N-i} \\
\sigma_{i, N-i}(Y, X, t) & \equiv \bar{H}_{i, N-i}^{(3, N)}(Y)+H_{i, N-i}^{(3, r, \leq K, N)}(Y, X, t) \varepsilon^{i} \mu^{N-i}
\end{aligned}
$$

Setting

$$
\Omega_{d}^{(N)}(Y ; \varepsilon, \mu) \equiv \omega(Y)+\sum_{i=1}^{N} \Omega_{i 0}(Y) \varepsilon^{i}+\sum_{j=1}^{N} \sum_{i=0}^{N-j} \Omega_{i j}(Y) \varepsilon^{i} \mu^{j},
$$

with $\Omega_{i 0} \equiv \bar{F}_{i 0}^{(3, \leq N)}(Y)$ and $\Omega_{i, N-i} \equiv \bar{F}_{i, N-i}^{(3, \leq N)}(Y)$, the normal form is finally given by

$$
\begin{aligned}
\dot{X} & =\Omega_{d}^{(N)}(Y ; \varepsilon, \mu)+\sum_{i=1}^{N} F_{i 0}^{(3, r, \leq K, \leq N)}(Y, X, t) \varepsilon^{i}+\sum_{j=1}^{N} \sum_{i=0}^{N-j} F_{i j}^{(3, r, \leq K, \leq N)}(Y, X, t) \varepsilon^{i} \mu^{j} \\
& +F_{N+1}(Y, X, t)+F^{(>K)}(Y, X, t) \\
\dot{Y} & =\sum_{i=1}^{N} G_{i 0}^{(3, r, \leq K, \leq N)}(Y, X, t) \varepsilon^{i}+G_{N+1}(Y, X, t)+G^{(>K)}(Y, X, t) \\
\dot{U} & =\sum_{i=1}^{N} H_{i 0}^{(3, r, \leq K, \leq N)}(Y, X, t) \varepsilon^{i}+H_{N+1}(Y, X, t)+H^{(>K)}(Y, X, t)
\end{aligned}
$$

where $F_{N+1}, G_{N+1}$ are $O_{N+1}(\varepsilon, \mu), H_{N+1}$ is order $O_{N+1}(\varepsilon)$ and $F^{(>K)}, G^{(>K)}, H^{(>K)}$ contain only terms with Fourier index greater than $K$. The normal form (8) is recovered with an obvious identification of the functions $F^{(r, \leq K)}, G^{(r, \leq K)}, H^{(r, \leq K)}$. The smallness requirements on $\varepsilon$, $\mu$, say $\varepsilon \leq \varepsilon_{0}, \mu \leq \mu_{0}$, are needed to guarantee the non-resonance condition (see (25), (30), (38), (43)) and the inversion of the transformations (see (20), (23), (28), (33), (39), (41)). 
The estimate (14) holds true, due to the definition of $\Omega_{d}^{(N)}$ in (44). The estimate (15) follows from the fact that (9) is close to the identity up to first order.

Due to the exponential decay of the Fourier coefficients (compare with Lemma B.1 of Appendix B), we can bound $G^{(>K)}$ for some $\tau_{0}>0$ as

$$
\left\|G^{(>K)}\right\|_{R_{0}, S_{0}} \leq \tilde{C}_{G} \lambda e^{-K \tau_{0}}
$$

for a suitable constant $\tilde{C}_{G}$. On the other hand we can bound $G_{N+1}$ in (45) as

$$
\left\|G_{N+1}\right\|_{R_{0}, S_{0}} \leq C_{G} \lambda^{N+1},
$$

for a suitable constant $C_{G}$. Finally, from the second of (45) we obtain:

$$
\begin{aligned}
\left\|\sum_{i=1}^{N} G_{i 0}^{(3, r, \leq K, \leq N)}(Y, X, t) \varepsilon^{j}\right\|_{R_{0}, S_{0}}+\left\|G_{N+1}\right\|_{R_{0}, S_{0}} & +\left\|G^{(>K)}\right\|_{R_{0}, S_{0}} \\
& \leq \lambda G+\lambda^{N+1} C_{G}+\lambda \tilde{C}_{G} e^{-K \tau_{0}}
\end{aligned}
$$

having defined $\lambda G$ as an upper bound of $\sup _{\varepsilon \leq \varepsilon_{0}}\left\|\sum_{i=1}^{N} G_{i 0}^{(3, r, \leq K, \leq N)}(Y, X, t) \varepsilon^{i}\right\|_{R_{0}, S_{0}}$. Choosing $N=\left[K \tau_{0} /|\log \lambda|\right]$, we obtain (16) and (17) with $C_{Y} \equiv C_{G}+\tilde{C}_{G}$. This concludes the proof of the Lemma.

Proof of the theorem. The distance between $y(t)$ and the initial condition $y(0)$ for $t \geq 0$ can be bounded by the sum of the following terms:

$$
\|y(t)-y(0)\| \leq\|y(t)-Y(t)\|+\|Y(t)-Y(0)\|+\|Y(0)-y(0)\| .
$$

By the estimate (15) of the Resonant Normal Form Lemma, one obtains

$$
\|y(t)-Y(t)\| \leq C_{p} \lambda, \quad\|y(0)-Y(0)\| \leq C_{p} \lambda
$$

By the second of (8) and by (16), one gets

$$
\begin{aligned}
\|Y(t)-Y(0)\| & \leq \int_{0}^{t}\left(\left\|G^{(r, \leq K)}\right\|_{R_{0}, S_{0}}+\left\|G_{N+1}\right\|_{R_{0}, S_{0}}+\left\|G^{(>K)}\right\|_{R_{0}, S_{0}}\right) d s \\
& \leq \lambda G t+C_{Y} \lambda^{N+1} t,
\end{aligned}
$$

which indicates that the action variation takes place on linear time scales due to the term $\lambda G t$, while exponential times are associated to the term $C_{Y} \lambda^{N+1} t$. We remark that $G=0$ corresponds to the absence of resonant terms in the normal form for $Y$. Notice that the case of non-resonant stability estimates given in [8] is recovered whenever also the resonant terms in 
the $X$ variable are zero. Let us start with the case $G=0$; for a suitable $\rho_{1}>0$ that we write as $\rho_{1}=C_{\rho} \lambda$ for some $C_{\rho}>0$, let

$$
t \leq \frac{C_{\rho}}{C_{Y}} e^{K \tau_{0}}
$$

Finally, setting $\rho_{0} \equiv\left(2 C_{p}+C_{\rho}\right) \lambda$, we obtain the following variation of the original action variables on exponential times:

$$
\|y(t)-y(0)\| \leq \rho_{0} \quad \text { for } t \leq C_{0} e^{K \tau_{0}},
$$

having defined $C_{0} \equiv C_{\rho} / C_{Y}$. This result is in agreement with statement $i$ ), once $G^{(r, \leq K)}$ is identified with $-\varepsilon p_{X}^{(\leq K)}$ (compare with (18)).

Next, we study the case $G \neq 0$; to this end, we compute the variation of the energy (i.e. the Lyapunov function, see e.g. [1]), which we intend to be defined as the energy function which is preserved whenever $\mu=0$. Let us write the normal form equations (45) using the following compact notation as in (18):

$$
\begin{aligned}
\dot{X} & =\omega(Y)+\varepsilon p_{Y}^{(\leq K)}(Y, X, t ; \varepsilon)+\mu s^{(\leq K)}(Y, X, t ; \varepsilon, \mu) \\
& +F^{(>K)}(Y, X, t ; \varepsilon, \mu)+F_{N+1}(Y, X, t ; \varepsilon, \mu) \\
\dot{Y} & =-\varepsilon p_{X}^{(\leq K)}(Y, X, t ; \varepsilon)+G^{(>K)}(Y, X, t ; \varepsilon, \mu)+G_{N+1}(Y, X, t ; \varepsilon, \mu) \\
\dot{U} & =-\varepsilon p_{t}^{(\leq K)}(Y, X, t ; \varepsilon)+H^{(>K)}(Y, X, t ; \varepsilon, \mu)+H_{N+1}(Y, X, t ; \varepsilon, \mu),
\end{aligned}
$$

where we have indicated also the dependence on the parameters and we have identified the functions as follows:

$$
\begin{aligned}
\varepsilon p_{Y}^{(\leq K)}(Y, X, t ; \varepsilon) & \equiv \sum_{i=1}^{N} \Omega_{i 0}(Y) \varepsilon^{i}+\sum_{i=1}^{N} F_{i 0}^{(3, r, \leq K, \leq N)}(Y, X, t) \varepsilon^{i} \\
\varepsilon p_{X}^{(\leq K)}(Y, X, t ; \varepsilon) & \equiv-\sum_{i=1}^{N} G_{i 0}^{(3, r, \leq K, \leq N)}(Y, X, t) \varepsilon^{i} \\
\varepsilon p_{t}^{(\leq K)}(Y, X, t ; \varepsilon) & \equiv-\sum_{i=1}^{N} H_{i 0}^{(3, r, \leq K, \leq N)}(Y, X, t) \varepsilon^{i} \\
\mu s^{(\leq K)}(Y, X, t ; \varepsilon, \mu) & \equiv \sum_{j=1}^{N} \sum_{i=0}^{N-j} F_{i j}^{(3, r, \leq K, \leq N)}(Y, X, t) \varepsilon^{i} \mu^{j}+\sum_{j=1}^{N} \sum_{i=0}^{N-j} \Omega_{i j}(Y) \varepsilon^{i} \mu^{j} .
\end{aligned}
$$

For $\mu=0$ equations (49) reduce to

$$
\begin{aligned}
\dot{X} & =\omega(Y)+\varepsilon p_{Y}^{(\leq K)}(Y, X, t ; \varepsilon)+F^{(>K)}(Y, X, t ; \varepsilon, 0)+F_{N+1}(Y, X, t ; \varepsilon, 0) \\
\dot{Y} & =-\varepsilon p_{X}^{(\leq K)}(Y, X, t ; \varepsilon)+G^{(>K)}(Y, X, t ; \varepsilon, 0)+G_{N+1}(Y, X, t ; \varepsilon, 0) \\
\dot{U} & =-\varepsilon p_{t}^{(\leq K)}(Y, X, t ; \varepsilon)+H^{(>K)}(Y, X, t ; \varepsilon, 0)+H_{N+1}(Y, X, t ; \varepsilon, 0) .
\end{aligned}
$$


Due to the Hamiltonian character of the equations of motion for $\mu=0$, there exist vector functions $A^{(>K)}, B_{N+1}$, such that

$$
\begin{gathered}
A_{Y}^{(>K)}=F^{(>K)}(Y, X, t ; \varepsilon, 0), \quad A_{X}^{(>K)}=-G^{(>K)}(Y, X, t ; \varepsilon, 0), \quad A_{t}^{(>K)}=-H^{(>K)}(Y, X, t ; \varepsilon, 0) \\
B_{N+1, Y}=F_{N+1}(Y, X, t ; \varepsilon, 0), \quad B_{N+1, X}=-G_{N+1}(Y, X, t ; \varepsilon, 0), \quad B_{N+1, t}=-H_{N+1}(Y, X, t ; \varepsilon, 0),
\end{gathered}
$$

so that we can recognize (50) as Hamilton's equations associated to the following Hamiltonian function in the extended phase space with $\dot{t}=1$ :

$$
\mathcal{H}(Y, X, U, t)=h_{00}(Y)+U+\varepsilon p^{(\leq K)}(Y, X, t)+A^{(>K)}(Y, X, t)+B_{N+1}(Y, X, t),
$$

where $h_{00}$ is such that $\frac{\partial h_{00}(Y)}{\partial Y}=\omega(Y)$. Let us fix the energy level $\mathcal{H}=E$ for some real constant $E$; taking into account the complete equations (49), we obtain that the variation of $E$ for $\mu \neq 0$ is given by (for simplicity we omit the arguments):

$$
\frac{d E}{d t}=\varepsilon \mu p_{X}^{(\leq K)} s^{(\leq K)}+C_{N+1}+D^{(>K)},
$$

with

$$
\begin{aligned}
C_{N+1} & \equiv \omega(Y) G_{N+1}+\varepsilon p_{Y}^{(\leq K)} G_{N+1}+\varepsilon p_{X}^{(\leq K)} F_{N+1} \\
& +\left(A_{Y}^{(>K)}+B_{N+1, Y}\right) G_{N+1}+B_{N+1, Y}\left(-\varepsilon p_{X}^{(\leq K)}+G^{(>K)}\right) \\
& +\left(A_{X}^{(>K)}+B_{N+1, X}\right) F_{N+1}+B_{N+1, X}\left(\omega+\varepsilon p_{Y}^{(\leq K)}+\mu s^{(\leq K)}+F^{(>K)}\right)+H_{N+1}+B_{N+1, t} \\
& \equiv \omega(Y) G^{(>K)}+\varepsilon p_{Y}^{(\leq K)} G^{(>K)}+\varepsilon p_{X}^{(\leq K)} F^{(>K)}+H^{(>K)} \\
& -\varepsilon p_{X}^{(\leq K)} A_{Y}^{(>K)}+A_{Y}^{(>K)} G^{(>K)}+\omega A_{X}^{(>K)}+\varepsilon p_{Y}^{(\leq K)} A_{X}^{(>K)} \\
& +\mu s^{(\leq K)} A_{X}^{(>K)}+A_{X}^{(>K)} F^{(>K)}+A_{t}^{(>K)}
\end{aligned}
$$

where now the functions $F_{N+1}, G_{N+1}, H_{N+1}, F^{(>K)}, G^{(>K)}, H^{(>K)}$ depend on $(Y, X, t ; \varepsilon, \mu)$. Denoting by $\Delta E \equiv E(t)-E(0)$, we obtain

$$
|\Delta E| \geq\left|\Delta h_{00}+\Delta U\right|-\left(\varepsilon\left\|\Delta p^{(\leq K)}\right\|_{R_{0}, S_{0}}+\left\|\Delta A^{(>K)}\right\|_{R_{0}, S_{0}}+\left\|\Delta B_{N+1}\right\|_{R_{0}, S_{0}}\right),
$$

where $\Delta h_{00}+\Delta U \equiv h_{00}(Y(t))-h_{00}(Y(0))+U(t)-U(0)$ and similarly for the other quantities. Recalling (51) and setting $h_{0} \equiv h_{00}+U$, we get

$$
\left|\Delta h_{0}\right| \leq|\Delta E|+\varepsilon\left\|\Delta p^{(\leq K)}\right\|_{R_{0}, S_{0}}+\left\|\Delta A^{(>K)}\right\|_{R_{0}, S_{0}}+\left\|\Delta B_{N+1}\right\|_{R_{0}, S_{0}},
$$

where

$$
|\Delta E| \leq\left|\frac{d E}{d t}\right| t \leq\left(\varepsilon \mu\left\|p_{X}^{(\leq K)}\right\|_{R_{0}, S_{0}}\left\|s^{(\leq K)}\right\|_{R_{0}, S_{0}}+\left\|C_{N+1}\right\|_{R_{0}, S_{0}}+\left\|D^{(>K)}\right\|_{R_{0}, S_{0}}\right) t .
$$


We denote by $m$ an upper bound on the Hessian of $h_{00}(y)$ and let $\tilde{m}$ be an upper bound of the Hessian in the normalized variables, which we can define as $\tilde{m} \equiv m+\left\|\frac{\partial^{3} h_{00}}{\partial y^{3}}\right\|_{r_{0}}\left\|D^{(y, N)}\right\|_{r_{0}, s_{0}}$, having expressed the link between new and old variables as $Y=y+D^{(y, N)}(y, x, t)$. Then, we have:

$$
\sup _{Y \in C_{r_{0}}(A)}\left\|\frac{\partial^{2} h_{00}(Y)}{\partial Y^{2}}\right\| \leq \tilde{m} .
$$

Assume that the frequency $\omega_{e}(y) \equiv(\omega(y), 1)$ is close to exact $\Lambda$-resonances (compare with [22]) by a quantity $\delta>0$, namely if $R_{\Lambda} \equiv\left\{\Omega \in \mathbb{R}^{\ell+1}: \Omega \cdot n=0\right.$ for all $\left.n \in \Lambda\right\}$, then $\min _{\Omega \in R_{\Lambda}}\left\|\omega_{e}(y)-\Omega\right\| \leq \delta$. Setting $Z \equiv(Y, U)$, assume that $|Z(t)-Z(0)| \leq r$ for some $r>0$ with $\delta+\tilde{m} r \leq R_{0}$. Let $\Pi_{\Lambda}$ be the orthogonal projection on $\Lambda$; by the mean value theorem we obtain

$$
\begin{aligned}
\left|\omega_{e} \cdot \Pi_{\Lambda} \Delta Z\right| & \leq\left\|\Pi_{\Lambda} \omega_{e}\right\|_{R_{0}}\|P \Delta Z\| \leq(\delta+\tilde{m} r)\|\Delta Z\| \\
\left|\omega_{e} \cdot\left(I d .-\Pi_{\Lambda}\right) \Delta Z\right| & \leq t\left\|\omega_{e}\right\|_{R_{0}}\left(\left\|G^{(>K)}\right\|_{R_{0}, S_{0}}+\left\|G_{N+1}\right\|_{R_{0}, S_{0}}+\left\|H^{(>K)}\right\|_{R_{0}, S_{0}}+\left\|H_{N+1}\right\|_{R_{0}, S_{0}}\right) .
\end{aligned}
$$

Moreover:

$$
\Delta h_{0}=\omega_{e} \cdot \Delta Z+\int_{0}^{1}(1-s) \frac{\partial^{2} h_{0}(Z(s))}{\partial Z^{2}} \Delta Z \cdot \Delta Z d s,
$$

so that, in the region where the $M$-convexity (2) holds, one has

$$
\frac{\tilde{M}}{2}\|\Delta Z\|^{2} \leq\left|\omega_{e} \cdot \Delta Z\right|+\left|\Delta h_{0}\right|,
$$

where (similarly to $\tilde{m}$ ) we can set $\tilde{M} \equiv M-\left\|\frac{\partial^{3} h_{00}}{\partial y^{3}}\right\|_{r_{0}}\left\|D^{(y, N)}(y, x, t)\right\|_{r_{0}, s_{0}}$, so that one has

$$
\frac{\partial^{2} h_{0}(Z)}{\partial Z^{2}} v \cdot v \geq \tilde{M}\|v\|^{2}, \quad \forall v \in \mathbb{R}^{\ell+1}
$$

Finally, we have

$$
\begin{aligned}
\frac{\tilde{M}}{2}\|\Delta Z\|^{2} & \leq(\delta+\tilde{m} r)\|\Delta Z\|+t\left\|\omega_{e}\right\|_{R_{0}}\left(\left\|G^{(>K)}\right\|_{R_{0}, S_{0}}+\left\|G_{N+1}\right\|_{R_{0}, S_{0}}\right. \\
& \left.+\left\|H^{(>K)}\right\|_{R_{0}, S_{0}}+\left\|H_{N+1}\right\|_{R_{0}, S_{0}}\right)+\varepsilon \mu\left\|p_{X}^{(\leq K)}\right\|_{R_{0}, S_{0}}\left\|s^{(\leq K)}\right\|_{R_{0}, S_{0}} t+\left\|C_{N+1}\right\|_{R_{0}, S_{0}} t \\
& +\left\|D^{(>K)}\right\|_{R_{0}, S_{0}} t+\varepsilon\left\|\Delta p^{(\leq K)}\right\|_{R_{0}, S_{0}}+\left\|\Delta A^{(>K)}\right\|_{R_{0}, S_{0}}+\left\|\Delta B_{N+1}\right\|_{R_{0}, S_{0}},
\end{aligned}
$$

which gives a bound on the norm of $\Delta Z$. Notice that $\left\|G^{(>K)}\right\|,\left\|H^{(>K)}\right\|$ and $\left\|\Delta A^{(>K)}\right\|$ are of order of $e^{-K \tau_{0}}$, namely of order $\lambda^{N}$ once we set $N$ such that $N=\left[K \tau_{0} /|\log \lambda|\right]$. We finally 
define the constants $C_{1}, C_{2}, C_{3}, C_{4}$ such that

$$
\begin{aligned}
\left\|\omega_{e}\right\|_{R_{0}}\left(\left\|G^{(>K)}\right\|_{R_{0}, S_{0}}\right. & \left.+\left\|G_{N+1}\right\|_{R_{0}, S_{0}}+\left\|H^{(>K)}\right\|_{R_{0}, S_{0}}+\left\|H_{N+1}\right\|_{R_{0}, S_{0}}\right) \\
+\left\|C_{N+1}\right\|_{R_{0}, S_{0}}+\left\|D^{(>K)}\right\|_{R_{0}, S_{0}} & \leq C_{1} \lambda^{N} \\
\left\|p_{X}^{(\leq K)}\right\|_{R_{0}, S_{0}}\left\|s^{(\leq K)}\right\|_{R_{0}, S_{0}} & \leq C_{2} \\
\left\|\Delta p^{(\leq K)}\right\|_{R_{0}, S_{0}} & \leq C_{3} \\
\left\|\Delta A^{(>K)}\right\|_{R_{0}, S_{0}}+\left\|\Delta B_{N+1}\right\|_{R_{0}, S_{0}} & \leq C_{4} \lambda^{N}
\end{aligned}
$$

With this setting we obtain:

$$
\frac{\tilde{M}}{2}\|\Delta Z\|^{2} \leq(\delta+\tilde{m} r)\|\Delta Z\|+C_{1} \lambda^{N} t+C_{2} \varepsilon \mu t+C_{3} \varepsilon+C_{4} \lambda^{N} .
$$

Based on the above formula and on $\|\Delta Y\| \leq\|\Delta Z\|$, we can draw the following conclusions:

1. for some $\rho_{1}>0,\|\Delta Y\| \leq \rho_{1}$ for $t$ of the order of $\lambda^{-N}$ if $C_{2}=0$, namely if $\left\|p_{X}^{(\leq K)}\right\|_{R_{0}, S_{0}}\left\|s^{(\leq K)}\right\|_{R_{0}, S_{0}}=0$, i.e. either $\left\|p_{X}^{(\leq K)}\right\|_{R_{0}, S_{0}}=0$ or $\left\|s^{(\leq K)}\right\|_{R_{0}, S_{0}}=0$

2. for some $\rho_{2}>0,\|\Delta Y\| \leq \rho_{2}$ for $t$ of the order of the minimum between $\lambda^{-N}$ and $(\varepsilon \mu)^{-1}$ if $C_{2} \neq 0$, i.e. $\left\|p_{x}^{(\leq K)}\right\|_{R_{0}, S_{0}}\left\|s^{(\leq K)}\right\|_{R_{0}, S_{0}} \neq 0$.

The two cases correspond to items $i$ ), $i i$ ) of the statement of the Theorem. More precisely, let us start with the case $C_{2}=0$, i.e. $p_{X}^{(\leq K)}=0$ or $s^{(\leq K)}=0$. Assuming that $\delta+\tilde{m} r<\alpha \tilde{M} r$, $C_{3} \varepsilon+C_{4} \lambda^{N}<\beta \tilde{M} r^{2}, t<\frac{1}{C_{1} \lambda^{N}} \gamma \tilde{M} r^{2}$ with $C_{1}>0$, for some positive constants $\alpha, \beta, \gamma$, under the assumption that $\|\Delta Z\| \leq r$ for some $r>0$, we obtain

$$
\frac{\tilde{M}}{2}\|\Delta Z\|^{2}<(\alpha+\beta+\gamma) \tilde{M} r^{2}
$$

namely

$$
\|\Delta Y\| \leq\|\Delta Z\|<\sqrt{2(\alpha+\beta+\gamma)} r \equiv \rho_{1}
$$

with $\rho_{1} \leq R_{0}$. Taking into account (48) and (15), one obtains item $i$ ) of the Theorem, namely

$$
\|y(t)-y(0)\| \leq 2 C_{p} \lambda+\rho_{1} \quad \text { for } t \leq T_{1} \equiv C_{0} e^{K \tau_{0}}
$$

with $C_{0} \equiv\left(\gamma \tilde{M} r^{2}\right) / C_{1}$.

Concerning item $i i)$, since $C_{2} \neq 0$ let $\sigma>0$ be such that for $t<\min \left(\frac{1}{C_{1} \lambda^{N}} \gamma \tilde{M} r^{2}, \frac{1}{C_{2} \varepsilon \mu} \sigma \tilde{M} r^{2}\right)$, one has

$$
\frac{\tilde{M}}{2}\|\Delta Z\|^{2}<(\alpha+\beta+\gamma+\sigma) \tilde{M} r^{2},
$$


namely

$$
\|\Delta Y\| \leq\|\Delta Z\|<\sqrt{2(\alpha+\beta+\gamma+\sigma)} r \equiv \rho_{2},
$$

with $\rho_{2} \leq R_{0}$. According to (48) and (15), we obtain that

$$
\|y(t)-y(0)\| \leq 2 C_{p} \lambda+\rho_{2} \quad \text { for } t \leq T_{2} \equiv \min \left(\frac{C_{0}}{\lambda^{N}}, \frac{C_{0}^{\prime}}{\varepsilon \mu}\right)
$$

with $C_{0} \equiv\left(\gamma \tilde{M} r^{2}\right) / C_{1}, C_{0}^{\prime} \equiv\left(\sigma \tilde{M} r^{2}\right) / C_{2}$.

When the $M$-convexity condition is violated (i.e. the second condition in (2)), by the assumption of quasi-convexity the first inequality in (2) must hold. Let $T$ be either $T_{1}$ or $T_{2}$ as in (54), (56) with $\left\|\omega_{e}\right\|_{R_{0}}$ replaced by

$$
C_{\omega} \equiv \sup _{\left\|Y-Y_{0}\right\| \leq \rho}\left\|\omega_{e}(Y)\right\|
$$

with $\rho$ being $\rho_{1}$ or $\rho_{2}$ as in (53), (55). Repeating the same argument as in [22], we assume that there exists an escape time $T_{e}$ such that $\|\Delta Z\|=\rho$ and we show that this implies the inequality

$$
\left|\omega_{e}(Y(s)) \cdot \Delta Z\right| \leq L\|\Delta Z\| \quad \forall 0 \leq s \leq 1 .
$$

Then, using the same argument as for the convex case, we conclude that $\|\Delta Z\|<\rho$, thus providing a contradiction. As before we have:

$$
\begin{aligned}
\left|\omega_{e}(Y(s)) \cdot P \Delta Z\right| & \leq(\delta+\tilde{m} r)\|\Delta Z\| \\
\left|\omega_{e}(Y(s)) \cdot(I d .-P) \Delta Z\right| & \leq C_{1} \lambda^{N} T .
\end{aligned}
$$

Then, we have

$$
\left|\omega_{e}(Y(s)) \cdot \Delta Z\right| \leq(\delta+\tilde{m} r)\|\Delta Z\|+C_{1} \lambda^{N} T .
$$

If $T=T_{1}, \rho=\rho_{1}$, we obtain

$$
\begin{aligned}
\left|\omega_{e}(Y(s)) \cdot \Delta Z\right| & \leq(\delta+\tilde{m} r)\|\Delta Z\|+C_{1} \lambda^{N} T_{1} \\
& <\alpha \tilde{M} r \rho_{1}+\gamma \tilde{M} r^{2} \\
& =\left(\frac{\alpha}{\sqrt{2(\alpha+\beta+\gamma)}}+\frac{\gamma}{2(\alpha+\beta+\gamma)}\right) \tilde{M} \rho_{1}^{2} \\
& \leq L\|\Delta Z\|,
\end{aligned}
$$

if

$$
\rho_{1} \leq \frac{L}{\left(\frac{\alpha}{\sqrt{2(\alpha+\beta+\gamma)}}+\frac{\gamma}{2(\alpha+\beta+\gamma)}\right) \tilde{M}} .
$$

If $T=T_{2}, \rho=\rho_{2}$, assume that $T=\left(\sigma \tilde{M} r^{2}\right) /\left(C_{2} \varepsilon \mu\right)$ (otherwise we recover the case $T=T_{1}$ ). Then,

$$
\left|\omega_{e}(Y(s)) \cdot \Delta Z\right| \leq(\delta+\tilde{m} r)\|\Delta Z\|+C_{1} \lambda^{N} \frac{\sigma \tilde{M} r^{2}}{C_{2} \varepsilon \mu}
$$


Being

$$
\frac{C_{1} \lambda^{N}}{C_{2} \varepsilon \mu} \leq \frac{\gamma}{\sigma}
$$

we obtain:

$$
\begin{aligned}
\left|\omega_{e}(Y(s)) \cdot \Delta Z\right| & <(\delta+\tilde{m} r)\|\Delta Z\|+\frac{\gamma}{\sigma} \sigma \tilde{M} r^{2} \\
& <\alpha \tilde{M} r\|\Delta Z\|+\gamma \tilde{M} r^{2} \\
& =\left(\frac{\alpha}{\sqrt{2(\alpha+\beta+\gamma+\sigma)}}+\frac{\gamma}{2(\alpha+\beta+\gamma+\sigma)}\right) \tilde{M} \rho_{2}^{2} \\
& \leq L\|\Delta Z\|,
\end{aligned}
$$

which is satisfied if the following condition holds:

$$
\rho_{2} \leq \frac{L}{\left(\frac{\alpha}{\sqrt{2(\alpha+\beta+\gamma+\sigma)}}+\frac{\gamma}{2(\alpha+\beta+\gamma+\sigma)}\right) \tilde{M}} .
$$

Remark 5. Since we do not claim the result for any $y_{0} \in A$, but only locally under the conditions (4) and (5), we do not need to cover the whole phase space and therefore we do not need the analysis of the geography of the resonances, as it is usually done (see, e.g., [22]).

\section{Applications of the normal forms}

As we have seen in the Theorem, the stability time depends on the expressions of the terms $p_{X}^{(\leq K)}$ and $s^{(\leq K)}$ appearing in the normal form equations, which represent, respectively, the conservative resonant part of the action variables and the dissipative resonant part pertaining to the angles, including the contribution of the modified frequency. In this Section we analyze several different examples, which well represent all possible situations which can be obtained with different choices of $p_{X}^{(\leq K)}$ and $s^{(\leq K)}$. We illustrate these models with a twofold goal: to provide examples of cases $i$ ) and $i i$ ) of the Theorem and to illustrate an explicit evaluation of the resonant normal form. Since we do not aim to obtain stability estimates, we limit ourselves to the computation of the normal form in the non-extended phase space, i.e. in the variables $x$ and $y$ only. The experiments performed in this Section will be validated by the theoretical results of Section 5, where the estimates of the Theorem will be applied, showing linear as well as exponential stability times.

All examples considered in the forthcoming Sections 4.1-4.4 will have the following simple form:

$$
\dot{x}=y+\mu f_{01}(x, t)
$$




$$
\dot{y}=-\varepsilon h_{10, x}(x, t)-\mu(y-\eta),
$$

where $f_{01}$ and $h_{10}$ are periodic functions. In this case it is easy to decide which of the conditions $i$ ) or $i i$ ) of the Theorem are satisfied. Since we shall not need to consider Fourier modes less or equal, or greater than $K$, we drop the superscript by writing $p_{X}, p_{Y}, s$ in place of $p_{X}^{(\leq K)}, p_{Y}^{(\leq K)}$, $s^{(\leq K)}$. Then, we can state that $p_{X}=0$ whenever the resonant part of $h_{10, x}$ is zero, otherwise $p_{X}$ is different from zero. Concerning the function $s$, we can state that if the resonant part of $f_{01}$ is not zero as well as if products of the form $\left(f_{01}\right)^{m}\left(h_{10, x}\right)^{n}$ with $0<m+n \leq N$ generate resonant terms or zero average terms of order $\mu$, then the function $s$ is different from zero. If the products $\left(f_{01}\right)^{m}\left(h_{10, x}\right)^{n}$ with $0<m+n \leq N$ do not generate resonant terms or zero average terms of order $\mu$, then $s=0$ up to the order $N$.

\subsection{Linear stability: case $p_{X} \neq 0, s \neq 0$}

We consider the one-dimensional, time-dependent vector field given by

$$
\begin{aligned}
& \dot{x}=y-\mu(\sin (x-t)+\sin (x)) \\
& \dot{y}=-\varepsilon(\sin (x-t)+\sin (x))-\mu(y-\eta) .
\end{aligned}
$$

Following the calculations of the proof of the Resonant Normal Form Lemma, the conservative transformation up to second order is defined by

$$
\begin{aligned}
& \psi_{10}(\tilde{y}, \tilde{x}, t)=\frac{\sin (\tilde{x})}{\tilde{y}} \\
& \psi_{20}(\tilde{y}, \tilde{x}, t)=\frac{\sin (2 \tilde{x}-t)}{2 \tilde{y}^{2}(2 \tilde{y}-1)}-\frac{\sin (t)}{2 \tilde{y}^{2}}-\frac{\sin (2 \tilde{x})}{8 \tilde{y}^{3}}
\end{aligned}
$$

while the dissipative contribution is given by

$$
\begin{aligned}
& \beta_{01}(Y, X, t)=0 \\
& \alpha_{01}(Y, X, t)=-\frac{\cos (X)}{Y} \\
& \beta_{11}(Y, X, t)=-\frac{\sin (2 X)}{4 Y^{2}}+\frac{\sin (t)}{Y} \\
& \alpha_{11}(Y, X, t)=-\frac{\cos (2 X-t)}{2 Y^{2}(2 Y-1)}-\frac{(2 Y+1) \cos (t)}{2 Y^{2}}+\frac{\cos (2 X)}{8 Y^{3}} \\
& \beta_{02}(Y, X, t)=0 \\
& \alpha_{02}(Y, X, t)=\frac{\sin (t)}{Y}+\frac{\sin (2 X)}{4 Y^{2}} .
\end{aligned}
$$


By choosing

$$
\eta(Y)=Y+\frac{\varepsilon}{2 Y}+O_{3}(\varepsilon, \mu)
$$

the normal form equations become

$$
\begin{aligned}
\dot{X} & =Y-\frac{\varepsilon^{2}}{2 Y^{3}}-\frac{\mu^{2}}{2 Y}-\mu \sin (X-t)+O_{3}(\varepsilon, \mu) \\
\dot{Y} & =-\varepsilon \sin (X-t)+O_{3}(\varepsilon, \mu),
\end{aligned}
$$

where we recognize that $p_{X}(Y, X, t)=\sin (X-t), p_{Y}(Y, X, t)=-\varepsilon /\left(2 Y^{3}\right), s(Y, X, t)=$ $-\sin (X-t)-\frac{\mu}{2 Y}$. The Hamiltonian function in the extended phase space with $U$ conjugated to time, associated to the normalized equations for $\mu=0$, is given by

$$
\mathcal{H}(Y, X, U, t)=\frac{Y^{2}}{2}+U+\frac{\varepsilon^{2}}{4 Y^{2}}-\varepsilon \cos (X-t)+O_{3}(\varepsilon, \mu) .
$$

Replacing the normalized equations into the total derivative of $\mathcal{H}$, one gets

$$
\frac{d \mathcal{H}(Y, X, U, t)}{d t}=-\frac{1}{2} \mu \varepsilon(1-\cos (2 X-2 t))+O_{3}(\varepsilon, \mu) .
$$

A typical orbit is shown in Figure 1, where we integrate the normal form equations for $\varepsilon=$ $10^{-3}$ and $\mu=10^{-3}$ with initial conditions $X(0)=0$ and $Y(0)=1+6 \sqrt{\varepsilon}$. The left panel of Figure 1 shows the lift of $(X, Y)$ to the universal coverage, while the middle panel shows the orbit back-transformed to the old variables $(x, y)$. The dynamics starts on a rotational regime and drifts downwards; then it spirals along librational invariant curves until reaching the attractor. The right panel of Figure 1 provides the variation of the derivative of the normal form Hamiltonian, which tends to zero as the orbit reaches the attractor. The behavior is justified by (58) as the resonance is approached.
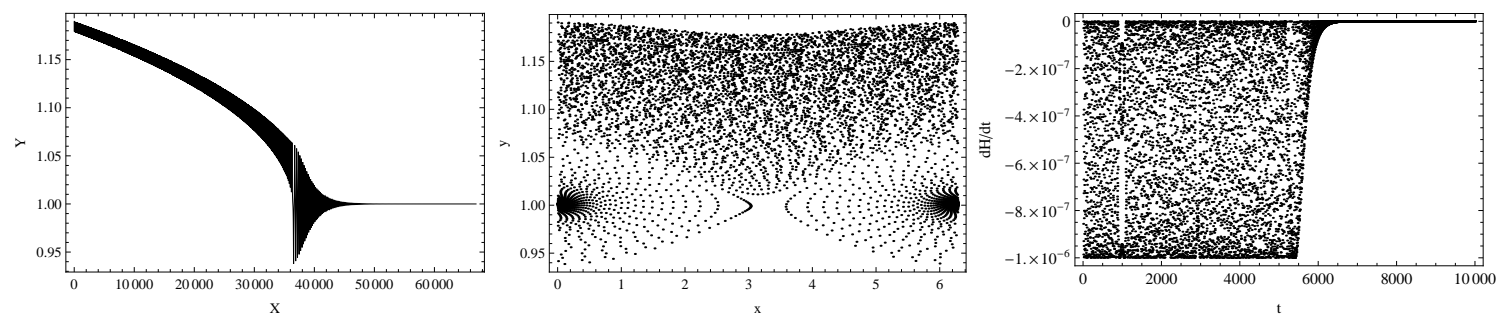

Figure 1: Case $p_{X} \neq 0, s \neq 0$ associated to (57) for $\varepsilon=10^{-3}, \mu=10^{-3}$ and for the initial conditions $X(0)=0, Y(0)=1+6 \sqrt{\varepsilon}$. Left: the lift of the normal form variables $(X, Y)$ to the universal coverage. Middle: the trajectory in the original variables $(x, y)$. Right: the variation of the derivative of the normalized Hamiltonian. 


\subsection{Linear stability at higher orders: case $p_{X} \neq 0, s \neq 0$}

We consider the vector field

$$
\begin{aligned}
& \dot{x}=y-\mu \sin (x) \\
& \dot{y}=-\varepsilon(\sin (x-t)+\sin (x))-\mu(y-\eta) .
\end{aligned}
$$

The conservative normal form is defined by

$$
\begin{aligned}
& \psi_{10}(\tilde{y}, \tilde{x}, t)=\frac{\sin (\tilde{x})}{\tilde{y}} \\
& \psi_{20}(\tilde{y}, \tilde{x}, t)=\frac{\sin (2 \tilde{x}-t)}{2 \tilde{y}^{2}(2 \tilde{y}-1)}-\frac{\sin (t)}{2 \tilde{y}^{2}}-\frac{\sin (2 \tilde{x})}{8 \tilde{y}^{3}} .
\end{aligned}
$$

The dissipative transformation becomes:

$$
\begin{aligned}
\beta_{01}(Y, X, t) & =0 \\
\alpha_{01}(Y, X, t) & =-\frac{\cos (X)}{Y} \\
\beta_{11}(Y, X, t) & =\frac{\sin (2 X-t)}{2 Y(2 Y-1)}+\frac{\sin (t)}{2 Y}-\frac{\sin (2 X)}{4 Y^{2}} \\
\alpha_{11}(Y, X, t) & =\frac{(1-3 Y) \cos (2 X-t)}{2 Y^{2}(2 Y-1)^{2}}+\frac{(1-Y) \cos (t)}{2 Y^{2}}+\frac{\cos (2 X)}{8 Y^{3}} \\
\beta_{02}(Y, X, t) & =0 \\
\alpha_{02}(Y, X, t) & =\frac{\sin (2 X)}{4 Y^{2}} .
\end{aligned}
$$

Higher normal form terms associated to (59) can be obtained in a similar way. For this model resonant terms occur at higher orders; for this reason we provide the following third order normal form equations:

$$
\begin{aligned}
\dot{X} & =Y-\frac{\varepsilon^{2}}{2 Y^{3}}-\frac{\mu^{2}}{2 Y}+\frac{\varepsilon^{3}(2-5 Y) \cos (X-t)}{2(1-2 Y)^{2} Y^{5}}-\frac{\varepsilon^{2} \mu\left(6 Y^{2}+2 Y-1\right) \sin (X-t)}{2(1-2 Y)^{2} Y^{3}} \\
& +\frac{1}{2} \varepsilon \mu^{2}\left(\frac{1}{Y^{2}}-\frac{2 Y}{(1-2 Y)^{2}}\right) \cos (X-t)+O_{4}(\varepsilon, \mu) \\
\dot{Y} & =-\varepsilon \sin (X-t)+\frac{\varepsilon^{3} \sin (X-t)}{8 Y^{5}-4 Y^{4}}+O_{4}(\varepsilon, \mu) .
\end{aligned}
$$

The $\eta$ is the same as in the previous Section, the Hamiltonian associated to the normal form in the extended phase space is given by

$$
\mathcal{H}(Y, X, U, t)=\frac{Y^{2}}{2}+U+\frac{\varepsilon^{2}}{4 Y^{2}}-\varepsilon \cos (X-t)+\frac{\varepsilon^{3} \cos (X-t)}{4 Y^{4}(2 Y-1)}+O_{4}(\varepsilon, \mu)
$$


while the derivative of the Hamiltonian becomes

$$
\frac{d \mathcal{H}(Y, X, U, t)}{d t}=-\frac{\varepsilon \mu^{2} \sin (X-t)}{2 Y}+O_{4}(\varepsilon, \mu) .
$$

The normal form produces a resonant term at third order. As a consequence, we observe a drift of the action variables, but on longer time scales.
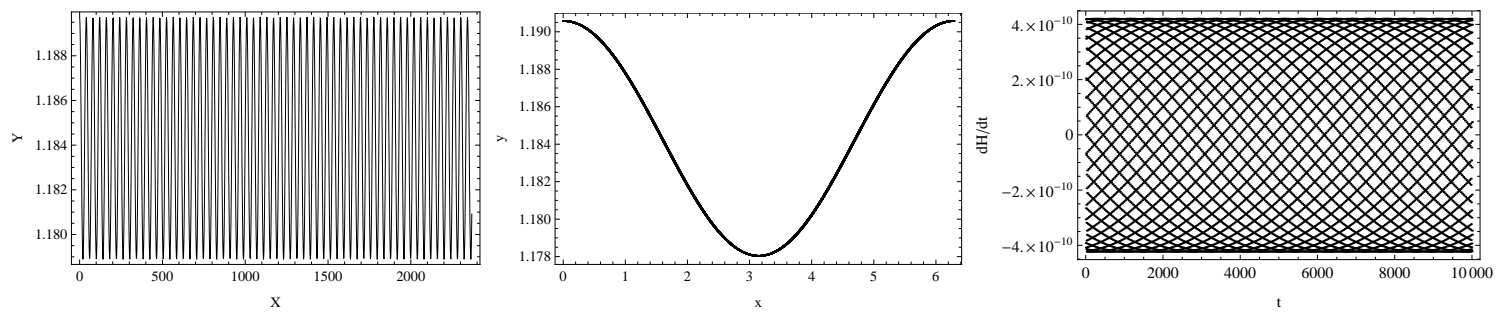

Figure 2: Case $p_{X} \neq 0, s \neq 0$ associated to (59) for $\varepsilon=10^{-3}, \mu=10^{-3}$ and for the initial conditions $X(0)=0, Y(0)=1+6 \sqrt{\varepsilon}$. Left: the lift of the normal form variables $(X, Y)$ to the universal coverage. Middle: the trajectory in the original variables $(x, y)$. Right: the variation of the derivative of the normalized Hamiltonian.

\subsection{Exponential stability: case $p_{X}=0, s \neq 0$}

We consider an example for which the normal form equations provide $p_{X}=0$, but $s \neq 0$. To this end, we modify the conservative part, so that the actions do not contain a resonant term at first order:

$$
\begin{aligned}
& \dot{x}=y-\mu(\sin (x-t)+\sin (x)) \\
& \dot{y}=-\varepsilon(\sin (x-6 t)+\sin (x))-\mu(y-\eta) .
\end{aligned}
$$

The conservative transformation to second order is given by

$$
\begin{aligned}
& \psi_{10}(\tilde{y}, \tilde{x}, t)=\frac{\sin (\tilde{x}-6 t)}{\tilde{y}-6}+\frac{\sin (\tilde{x})}{\tilde{y}} \\
& \psi_{20}(\tilde{y}, \tilde{x}, t)=-\frac{\sin (2 \tilde{x})}{8 \tilde{y}^{3}}-\frac{\sin (2 \tilde{x}-6 t)}{4 \tilde{y}^{3}-36 \tilde{y}^{2}+72 \tilde{y}}-\frac{\sin (2 \tilde{x}-12 t)}{8(\tilde{y}-6)^{3}}+\frac{\sin (6 t)}{72 \tilde{y}-12 \tilde{y}^{2}} .
\end{aligned}
$$

The dissipative transformation to second order takes the form

$$
\begin{aligned}
\beta_{01}(Y, X, t) & =0 \\
\alpha_{01}(Y, X, t) & =-\frac{\cos (X)}{Y}
\end{aligned}
$$




$$
\begin{aligned}
\beta_{11}(Y, X, t) & =-\frac{\sin (2 X-7 t)}{4 Y^{2}-38 Y+84}-\frac{\sin (2 X-6 t)}{4 Y^{2}-36 Y+72}-\frac{\sin (2 X)}{4 Y^{2}} \\
& +\frac{\sin (2 X-t)}{2 Y-4 Y^{2}}+\frac{\sin (t)}{2 Y}+\frac{\sin (5 t)}{10(Y-6)}+\frac{\sin (6 t)}{12(Y-6)} \\
\alpha_{11}(Y, X, t) & =\frac{\cos (2 X)}{8 Y^{3}}+\frac{\cos (2 X-7 t)}{2(7-2 Y)^{2}(Y-6)}+\frac{\cos (2 X-6 t)}{8(Y-6)(Y-3)^{2}} \\
& +\frac{\cos (2 X-t)}{2(1-2 Y)^{2} Y}-\frac{(Y+2) \cos (t)}{2 Y^{2}}-\frac{(Y-16) \cos (5 t)}{50(Y-6)^{2}}-\frac{(Y-18) \cos (6 t)}{72(Y-6)^{2}} \\
\beta_{02}(Y, X, t) & =0 \\
\alpha_{02}(Y, X, t) & =\frac{\sin (2 X)}{4 Y^{2}}+\frac{\sin (t)}{Y} .
\end{aligned}
$$

The resulting normal form up to second order becomes

$$
\begin{aligned}
\dot{X} & =Y-\frac{\varepsilon^{2}}{2(Y-6)^{3}}-\frac{\varepsilon^{2}}{2 Y^{3}}-\frac{\mu^{2}}{2 Y}-\mu \sin (X-t)+O_{3}(\varepsilon, \mu) \\
\dot{Y} & =O_{3}(\varepsilon, \mu)
\end{aligned}
$$

whereas the drift function is given by

$$
\eta(Y)=Y+\frac{\varepsilon}{2 Y}+O_{3}(\varepsilon, \mu)
$$

The Hamiltonian function in normalized variables corresponding to $\mu=0$ in the extended phase space turns out to be

$$
\mathcal{H}(Y, X, U, t)=\frac{Y^{2}}{2}+T+\frac{\left(Y^{2}-6 Y+18\right) \varepsilon^{2}}{2(Y-6)^{2} Y^{2}}+O_{3}(\varepsilon, \mu) ;
$$

the time derivative of the Hamiltonian under the dissipative flow becomes

$$
\frac{d \mathcal{H}(Y, X, U, t)}{d t}=O_{3}(\varepsilon, \mu),
$$

which shows the preservation of the energy up to the third order. Figure 3 displays the behavior of the lift of $(X, Y)$ to the universal covering, the plot in the original variables and the graph of the derivative of the Hamiltonian versus time. The result shows that the dynamics takes place on an adiabatic quasi-periodic solution, which is consistent with the theoretical expectation.

\subsection{Exponential stability: case $p_{X} \neq 0, s=0$}

As an example which generates a normal form with $p_{X} \neq 0, s=0$, we consider the differential equations

$$
\begin{aligned}
& \dot{x}=y-\mu \sin (6 t) \\
& \dot{y}=-\varepsilon(\sin (x-t)+\sin (x))-\mu(y-\eta) .
\end{aligned}
$$



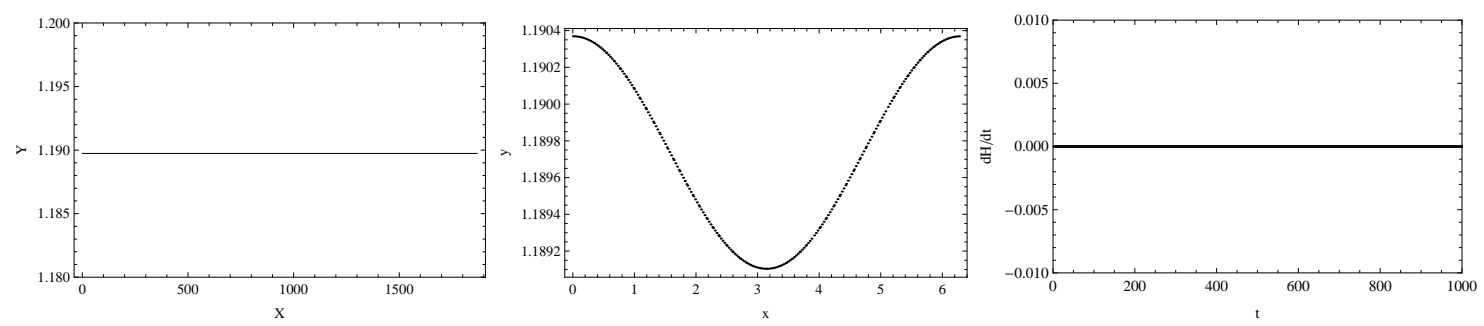

Figure 3: Case $p_{X}=0, s \neq 0$ associated to (60) for $\varepsilon=10^{-3}, \mu=10^{-3}$ and for the initial conditions $X(0)=0, Y(0)=1+6 \sqrt{\varepsilon}$. Left: the lift of the normal form variables $(X, Y)$ to the universal coverage. Middle: the trajectory in the original variables $(x, y)$. Right: the variation of the derivative of the normalized Hamiltonian.

The conservative transformation is given by

$$
\begin{aligned}
& \psi_{10}(\tilde{y}, \tilde{x}, t)=\frac{\sin (\tilde{x})}{\tilde{y}} \\
& \psi_{20}(\tilde{y}, \tilde{x}, t)=-\frac{\sin (2 \tilde{x})}{8 \tilde{y}^{3}}-\frac{\sin (2 \tilde{x}-t)}{2 \tilde{y}^{2}-4 \tilde{y}^{3}}-\frac{\sin (t)}{2 \tilde{y}^{2}}
\end{aligned}
$$

while the dissipative transformation takes the form

$$
\begin{aligned}
& \beta_{01}(Y, X, t)=0 \\
& \alpha_{01}(Y, X, t)=-\frac{1}{6} \cos (6 t) \\
& \beta_{11}(Y, X, t)=-\frac{\sin (X-6 t)}{12 Y-2 Y^{2}}-\frac{\sin (X-7 t)}{84-12 Y}+\frac{\sin (X+5 t)}{12 Y+60}-\frac{\sin (X+6 t)}{2 Y(Y+6)} \\
& \alpha_{11}(Y, X, t)=\frac{(3-Y) \cos (X-6 t)}{(Y-6)^{2} Y^{2}}+\frac{(Y+3) \cos (X+6 t)}{Y^{2}(Y+6)^{2}}-\frac{\cos (X-7 t)}{12(Y-7)^{2}}-\frac{\cos (X+5 t)}{12(Y+5)^{2}} \\
& \beta_{02}(Y, X, t)=0 \\
& \alpha_{02}(Y, X, t)=0 .
\end{aligned}
$$

The normal form equations are given by

$$
\begin{aligned}
\dot{X} & =Y-\frac{\varepsilon^{2}}{2 Y^{3}}+O_{3}(\varepsilon, \mu) \\
\dot{Y} & =-\varepsilon \sin (X-t)+O_{3}(\varepsilon, \mu),
\end{aligned}
$$

with the drift function provided by $\eta(Y, X, t)=Y-\frac{1}{144} \varepsilon \mu \sin (X-t)+O_{3}(\varepsilon, \mu)$. Note that we produce linear conservative resonant terms in the actions, but no resonant dissipative terms in 
the angles. The Hamiltonian function associated to the normal form equations in the extended phase space becomes

$$
\mathcal{H}(Y, X, U, t)=\frac{Y^{2}}{2}+T+\frac{\varepsilon^{2}}{4 Y^{2}}-\varepsilon \cos (X-t)+O_{3}(\varepsilon, \mu),
$$

while the time derivative of the Hamiltonian flow becomes;

$$
\frac{d \mathcal{H}(Y, X, U, t)}{d t}=O_{3}(\varepsilon, \mu)
$$

yielding the preservation of the Hamiltonian up to the normalization order. Figure 4 shows the behavior of the lift of $(X, Y)$ to the universal covering, the graph in the original variables and the plot of the derivative of the Hamiltonian versus time. Also in this case, the result shows that the dynamics takes place on an adiabatic quasi-periodic solution, which is consistent with the theoretical expectation.
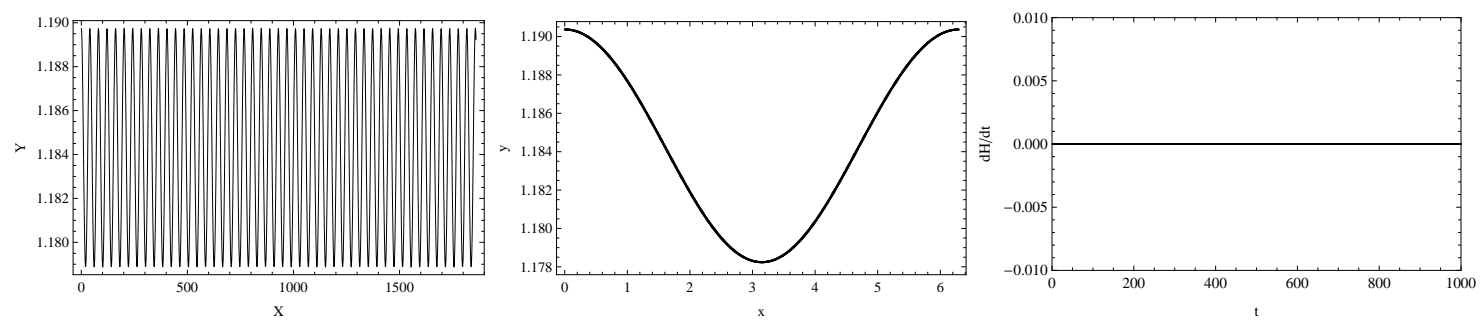

Figure 4: Case $p_{X} \neq 0, s=0$ associated to (61) for $\varepsilon=10^{-3}, \mu=10^{-3}$ and for the initial conditions $X(0)=0, Y(0)=1+6 \sqrt{\varepsilon}$. Left: the lift of the normal form variables $(X, Y)$ to the universal coverage. Middle: the trajectory in the original variables $(x, y)$. Right: the variation of the derivative of the normalized Hamiltonian.

\section{Application of the stability estimates}

In this Section we implement the Theorem to obtain estimates on the variation of the actions as given in Section 3. Let us fix the initial data as well as $r_{0}, s_{0}$ (and related domain's parameters), $K, \delta$. We assume that the frequency satisfies (4), (5) with $a$ determined by (5). The smallness conditions on the parameters $\varepsilon, \mu$, say $\varepsilon \leq \varepsilon_{0}, \mu \leq \mu_{0}$, come from (20), (23), (25), (28), (30), (33), (38), (39), (41), (43).

We define the constants $\tilde{C}_{G}$ and $C_{G}$ as in (46), (47) and we set $C_{Y} \equiv C_{G}+\tilde{C}_{G}$. We recall that $\tau_{0}, N, K$ are related by the expression

$$
\tau_{0} \equiv \frac{N}{K}|\log \lambda|
$$




\begin{tabular}{lllll}
\hline \hline- & Sec. 4.1 & Sec. 4.2 & Sec. 4.3 & Sec. 4.4 \\
$\varepsilon_{0}$ & $6 . \cdot 10^{-5}$ & $6 . \cdot 10^{-5}$ & $6 . \cdot 10^{-5}$ & $6 . \cdot 10^{-5}$ \\
$\mu_{0}$ & $6 . \cdot 10^{-5}$ & $6 . \cdot 10^{-5}$ & $6 . \cdot 10^{-5}$ & $1.9 \cdot 10^{-4}$ \\
$\tau_{0}$ & 1.458 & 1.458 & 1.458 & 1.285 \\
$C_{Y}$ & $3.16 \cdot 10^{1}$ & $3.428 \cdot 10^{1}$ & $1.714 \cdot 10^{1}$ & 1.087 \\
$C_{p}$ & 1.052 & 1.052 & 1.265 & $3.323 \cdot 10^{-1}$ \\
$C_{1}$ & $2.117 \cdot 10^{-3}$ & $2.233 \cdot 10^{-3}$ & $1.359 \cdot 10^{-3}$ & $2.158 \cdot 10^{-4}$ \\
$C_{2}$ & $5.056 \cdot 10^{-3}$ & $3.059 \cdot 10^{-5}$ & 0 & 0 \\
$C_{3}$ & 2.01 & 2.01 & $3.208 \cdot 10^{-5}$ & 2.01 \\
$C_{4}$ & $3.292 \cdot 10^{-5}$ & $3.292 \cdot 10^{-5}$ & $1.006 \cdot 10^{-5} \cdot 3.283 \cdot 10^{-6}$ \\
$\|\Delta Y\|$ & $2.408 \cdot 10^{-2}$ & $2.408 \cdot 10^{-2}$ & $9.369 \cdot 10^{-3}$ & $2.408 \cdot 10^{-2}$ \\
$\|\Delta y\|$ & $2.421 \cdot 10^{-2}$ & $2.421 \cdot 10^{-2}$ & $9.521 \cdot 10^{-3}$ & $2.421 \cdot 10^{-2}$ \\
$T$ & $2.692 \cdot 10^{5}$ & $4.43 \cdot 10^{7}$ & $1.699 \cdot 10^{10}$ & $3.309 \cdot 10^{9}$ \\
\hline \hline
\end{tabular}

Table 1: The main quantities of the Theorem for the examples of Section 4 from the remainder of a third order normal form. The parameters and initial conditions for all columns are: $x_{0}=0$, $y_{0}=1.01, r_{0}=0.05, \tilde{r}_{0}=4.9 \cdot 10^{-2}, \tilde{r}_{0}^{\prime}=2.45 \cdot 10^{-2}, R_{0}=2.4 \cdot 10^{-2}, s_{0}=0.1, \tilde{s}_{0}=5 \cdot 10^{-3}$, $S_{0}=2.5 \cdot 10^{-3}, K=20, \delta=0.01$.

From (15) we determine $C_{p}$, while the constants $C_{1}, C_{2}, C_{3}, C_{4}$ are computed as in (52). Table 1 provides the main quantities involved in the Theorem through the application of a third order normal form in the extended phase space. In particular, it provides the variation $\|\Delta Y\|$ of the normalized variables, the variation $\|\Delta y\| \equiv\|y(t)-y(0)\|$ of the original variables and the stability time $T$, which perfectly agrees with the theoretical result (linear or exponential stability time) of the Theorem.

The results have been validated by a numerical integration of the equations of motion. Due to computer limitations, for the cases described in Sections 4.3 and 4.4 we had to stop to a time at most equal to $10^{8}$. Up to such integration times the numerical results are in full agreement with the analytical results. 


\section{Appendix A}

We briefly review the conditions which must be satisfied by the parameters $\varepsilon, \mu$, so that the transformation from original to intermediate variables, as well as that from intermediate to final variables can be inverted; moreover, we provide conditions on the parameters so that the non-resonance conditions in the intermediate and final variables are satisfied. Compare also with [8] and [12].

\subsection{Inversion of the conservative transformation}

With reference to (31), we invert the first transformation as

$$
x=\tilde{x}+\Gamma^{(x, N)}(\tilde{y}, \tilde{x}, t)
$$

provided that

$$
70\left\|\psi_{y}^{(N)}\right\|_{\tilde{r}_{0}, s_{0}} e^{2 s_{0}} \delta_{0}^{-1}<1
$$

with

$$
\left\|\Gamma^{(x, N)}\right\|_{\tilde{r}_{0}, \tilde{s}_{0}} \leq\left\|\psi_{y}^{(N)}\right\|_{\tilde{r}_{0}, s_{0}} .
$$

for $\tilde{r}_{0}<r_{0}, \delta_{0}<s_{0}, \tilde{s}_{0} \equiv s_{0}-\delta_{0}$.

\subsection{Non-resonance condition after the conservative transformation}

Taking into account (5), we want that the non-resonance condition is satisfied in the intermediate variables, say for $a>0$ :

$$
|\omega(\tilde{y}) \cdot k+m|>\frac{a}{2}, \quad|k|+|m| \leq K .
$$

The second of (31) can be inverted as

$$
\tilde{y}=y+\varepsilon R^{(N)}(y, x, t),
$$

for a suitable function $R^{(N)}$ provided

$$
70\left\|\psi_{x}^{(N)}\right\|_{\tilde{r}_{0}, s_{0}} \frac{1}{\tilde{r}_{0}-\tilde{r}_{0}^{\prime}}<1
$$

for $\tilde{r}_{0}^{\prime}<\tilde{r}_{0}$ with

$$
\varepsilon\left\|R^{(N)}\right\|_{\tilde{r}_{0}^{\prime}, s_{0}} \leq\left\|\psi_{x}^{(N)}\right\|_{\tilde{r}_{0}, s_{0}} .
$$

Then we have

$$
|\omega(\tilde{y}) \cdot k+m| \geq \frac{a}{2},
$$

if

$$
\varepsilon \leq \frac{a}{2 K\left\|R^{(N)}\right\|_{\tilde{r}_{0}^{\prime}, s_{0}}\left\|\omega_{y}\right\|_{r_{0}}} .
$$




\subsection{Inversion of the dissipative transformation}

With reference to (12), the first equation can be inverted provided

$$
70\left\|\alpha^{(N)}\right\|_{\tilde{r}_{0}, \tilde{s}_{0}} e^{2 \tilde{s}_{0}} \tilde{\delta}_{0}^{-1}<1,
$$

where $\tilde{\delta}_{0}<\tilde{s}_{0}$. Inverting the equation as

$$
\tilde{x}=X+A^{(x, N)}(\tilde{y}, X, t),
$$

we have

$$
\left\|A^{(x, N)}\right\|_{\tilde{r}_{0}, \tilde{s}_{0}-\tilde{\delta}_{0}} \leq\left\|\alpha^{(N)}\right\|_{\tilde{r}_{0}, \tilde{s}_{0}} .
$$

Thus we invert the second of (12) as

$$
\tilde{y}=Y+\Delta^{(y, N)}(Y, X, t),
$$

provided

$$
70\left\|A^{(y, N)}\right\|_{\tilde{r}_{0}, S_{0}} \frac{1}{\tilde{r}_{0}-R_{0}}<1
$$

with $S_{0}<\tilde{s}_{0}-\tilde{\delta}_{0}, R_{0}<\tilde{r}_{0}$, being

$$
\left\|\Delta^{(y, N)}\right\|_{R_{0}, S_{0}} \leq\left\|A^{(y, N)}\right\|_{\tilde{r}_{0}, S_{0}} .
$$

Notice that $A^{(y, N)}$ can be bounded as

$$
\left\|A^{(y, N)}\right\|_{\tilde{r}_{0}, S_{0}} \leq\left\|\beta^{(N)}\right\|_{\tilde{r}_{0}, \tilde{s}_{0}}+\left\|\beta_{x}^{(N)}\right\|_{\tilde{r}_{0}, \tilde{s}_{0}}\left\|A^{(x, N)}\right\|_{\tilde{r}_{0}, S_{0}} .
$$

Similar for the third equation in (12).

\subsection{Non-resonance condition after the dissipative transformation}

We now turn to the fulfillment of the non-resonant condition in the new set of variables

$$
|\omega(Y) \cdot k+m|>0, \quad|k|+|m| \leq K .
$$

Through the transformation

$$
Y=\tilde{y}+\beta^{(N)}(\tilde{y}, \tilde{x}, t ; \varepsilon, \mu)
$$

and using (63) one finds

$$
|\omega(Y) \cdot k+m| \geq \frac{a}{4}
$$

provided that

$$
K\left\|\omega_{y}\right\|_{r_{0}}\left\|\beta^{(N)}\right\|_{\tilde{r}_{0}, \tilde{s}_{0}}<\frac{a}{4}
$$




\section{Appendix B}

From properties of analytic functions one can prove the following result (see also [8]) on the decay of the tail of the Fourier series.

Lemma B.1. Let $f=f(y, x, t)$ be an analytic function on the domain $C_{r_{0}}(A) \times C_{s_{0}}\left(\mathbb{T}^{\ell+1}\right)$. Let $f^{>K}(y, x, t) \equiv \sum_{(j, m) \in \mathbb{Z}^{\ell+1},|j|+|m|>K} \hat{f}_{j m}(y) e^{i(j \cdot x+m t)}$ and let $0<\sigma_{0}<s_{0}$. Then, there exists a constant $C_{a} \equiv C_{a}\left(\sigma_{0}, K\right)$, such that

$$
\left\|f^{>K}\right\|_{r_{0}, s_{0}} \leq C_{a}\|f\|_{r_{0}, s_{0}+\sigma_{0}} e^{-(K+1) \sigma_{0}},
$$

with

$$
C_{a} \equiv e^{(K+1) \frac{\sigma_{0}}{2}}\left(\frac{1+e^{-\frac{\sigma_{0}}{2}}}{1-e^{-\frac{\sigma_{0}}{2}}}\right)^{\ell+1}
$$

Acknowledgments. We are grateful to Luca Biasco, Enrico Valdinoci and Jean-Christophe Yoccoz for interesting discussions and suggestions. We acknowledge the grants ASI "Studi di Esplorazione del Sistema Solare" and PRIN 2007B3RBEY "Dynamical Systems and Applications" of MIUR.

\section{References}

[1] V.I. Arnold (editor), Encyclopaedia of Mathematical Sciences, Dynamical Systems III, Springer-Verlag 3 (1988)

[2] G. Benettin, L. Galgani, A. Giorgilli, A Proof of Nekhoroshev's Theorem for the stability times in nearly integrable Hamiltonian systems, Celest. Mech. Dyn. Astron. 37, 1-25 (1985)

[3] A. Bounemoura, J.-P. Marco, Improved exponential stability for near-integrable quasiconvex Hamiltonians, Nonlinearity 24, 97-112 (2011)

[4] R. Calleja, A. Celletti, R. de la Llave, KAM theory for conformally symplectic systems, Preprint 2011, available on the Mathematical Physics Preprint Archive: mp_arc 11-188

[5] A. Celletti, Stability and Chaos in Celestial Mechanics, Springer-Praxis 2010, XVI, 264 pp., Hardcover ISBN: 978-3-540-85145-5

[6] A. Celletti, L. Ferrara, An application of the Nekhoroshev theorem to the restricted threebody problem, Cel. Mech. Dyn. Astr. 64, 261-272 (1996) 
[7] A. Celletti, A. Giorgilli, On the stability of the Lagrangian points in the spatial restricted problem of three bodies, Cel. Mech. Dyn. Astr. 50, 31-58 (1991)

[8] A. Celletti, C. Lhotka, Stability bounds for exponential times in nearly-Hamiltonian systems with non-resonant frequency, Preprint 2011

[9] L. Chierchia, C. Falcolini, A direct proof of a theorem by Kolmogorov in Hamiltonian systems, Annali Scuola Normale Sup. Pisa, Scienze Fisiche e Matematiche, XXI Fasc. 4, 541-593 (1994)

[10] A. Delshams, A. Guillamon, J.T. Lazaro, A pseudo-normal form for planar vector fields, Qualitative theory of Dynamical Systems 3, 51-82 (2002)

[11] G. Gallavotti, Twistless KAM tori, Comm. Math. Phys. 164, n. 1, 145-156 (1994)

[12] G. Gallavotti, The Elements of Mechanics Springer-Verlag (1983)

[13] A. Giorgilli, Effective stability in Hamiltonian systems in the light of Nekhoroshev's theorem, Integrable Systems and Applications, Springer-Verlag, Berlin, New York, 142 153 (1989)

[14] A. Giorgilli, A. Delshams, E. Fontich, L. Galgani, C. Simó, Effective stability for a Hamiltonian system near an elliptic equilibrium point, with an application to the restricted three body problem, J. Diff. Eq. 77, 167-198 (1989)

[15] A. Giorgilli, Ch. Skokos, On the stability of the Trojan asteroids, Astron. Astroph. 317, 254-261 (1997)

[16] A. Giorgilli, E. Zehnder, Exponential Stability for time dependent potentials, ZAMP 43, n. $5,827-855$ (1992)

[17] I.P. Goulden, D.M. Jackson, Combinatorial Enumeration, Wyley Interscience Series in Discrete Math. (1983)

[18] G. Ioos, E. Lombardi, Polynomial normal forms with exponentially small remainder for analytic vector fields, J. Diff. Eq. 212, 1-61 (2005)

[19] C. Lhotka, C. Efthymiopoulos, R. Dvorak, Nekhoroshev stability at $L_{4}$ or $L_{5}$ in the elliptic-restricted three-body problem - application to Trojan asteroids, MNRAS 384, no. 3, 1165-1177 (2008)

[20] N.N. Nekhoroshev, An exponential estimate of the stability time of near-integrable Hamiltonian systems, Russ. Math. Surveys 32, no. 6, 1-65 (1977) 
[21] N.N. Nekhoroshev, Exponential estimates of the stability time of near-integrable Hamiltonian systems 2, Trudy Sem. Petrovos. 5, 5-50, 1979

[22] J. Pöschel, Nekhoroshev's estimates for quasi-convex Hamiltonian systems, Math. Z. 213, 187-216 (1993) 